\title{
Quantum propensities in the brain cortex and free will
}

\author{
Danko D. Georgiev ${ }^{\mathrm{a}, *}$ \\ ${ }^{a}$ Institute for Advanced Study, 30 Vasilaki Papadopulu Str., Varna 9010, Bulgaria
}

\begin{abstract}
Capacity of conscious agents to perform genuine choices among future alternatives is a prerequisite for moral responsibility. Determinism that pervades classical physics, however, forbids free will, undermines the foundations of ethics, and precludes meaningful quantification of personal biases. To resolve that impasse, we utilize the characteristic indeterminism of quantum physics and derive a quantitative measure for the amount of free will manifested by the brain cortical network. The interaction between the central nervous system and the surrounding environment is shown to perform a quantum measurement upon the neural constituents, which actualize a single measurement outcome selected from the resulting quantum probability distribution. Inherent biases in the quantum propensities for alternative physical outcomes provide varying amounts of free will, which can be quantified with the expected information gain from learning the actual course of action chosen by the nervous system. For example, neuronal electric spikes evoke deterministic synaptic vesicle release in the synapses of sensory or somatomotor pathways, with no free will manifested. In cortical synapses, however, vesicle release is triggered indeterministically with probability of 0.35 per spike. This grants the brain cortex, with its over 100 trillion synapses, an amount of free will exceeding 96 terabytes per second. Although reliable deterministic transmission of sensory or somatomotor information ensures robust adaptation of animals to their physical environment, unpredictability of behavioral responses initiated by decisions made by the brain cortex is evolutionary advantageous for avoiding predators. Thus, free will may have a survival value and could be optimized through natural selection.
\end{abstract}

Keywords: choice, conscious agent, determinism, information gain, quantum indeterminism, synapse

\section{Highlights}

- Free will is the capacity of conscious agents to choose a future course of action among several available physical alternatives.

- Expected information gain from learning the choice of a conscious agent provides a quantitative measure of free will.

- Quantum indeterminism supports varying amounts of free will exercised in different quantum measurement contexts.

- Probabilistic release of synaptic vesicles from cortical synapses grants on average 0.934 bits of free will per synapse per spike.

- Unpredictability of animal behavior provides a survival advantage and allows for evolutionary optimization of manifested free will.

\footnotetext{
${ }^{*}$ Corresponding author

Email address: danko.georgiev@mail.bg (Danko D. Georgiev)
} 


\section{Introduction}

We are conscious beings who feel in control of their future actions [1]. Good choices make us happy and elevate well-being, whereas bad choices make us regret about missed opportunities and precipitate suffering. Importantly, the choices that we make on a daily basis impact not only our lives, but also the lives of others who depend on us. Consequently, the capacity of being able to choose freely, comes with a moral responsibility and accountability for our own actions [2-4]. Thus, free will appears to be a main prerequisite for personal fulfillment, through undertaking steps toward achievement of individual life goals $[5,6]$, and the construction of a moral and just society, through legislation of civil law that guarantees equal basic rights to all society members [7].

Freedom is a basic human right and everyone instinctively strives for a life without external coercion. We enjoy being free and the existence of free will is persistently corroborated by our own introspective testimony, e.g., whenever we decide to move our arms or legs [1]. Thus, it may come as a surprise that a neuroscientist could even consider challenging the veracity of free will, which our conscious experiences reveal to us. Moreover, regardless of how impeccable the logical reasoning against free will may be, in ordinary circumstances such a directly verifiable fact should be impervious to any argumentation that goes against it. Instead, arriving at a contradiction should be considered as a proof against the veracity of the set of initial assumptions upon which the logical reasoning is based [1]. Nonetheless, it appears that what would be considered abnormal in ordinary circumstances is accepted as quite normal in the philosophy of free will [8]. From the deterministic nature of physical laws in classical mechanics [9], it has been often concluded that free will is impossible and we believe in an illusion [10-13]. Because the physical laws hold true at all times and do not evolve, it appears that free will cannot evolve too, namely, either we have free will or we do not, by virtue of physical laws. Indeed, if physical laws always forbid the existence of free will, there should be no sense in which we acquire free will gradually through natural selection. Furthermore, the concept of free will, defined as the capacity of agents to choose a course of action among at least two alternative future possibilities, appears to be a rough qualitative statement that is unable to account for the possible presence of inherent biases in favor of some of the available choices. Here, we will show that free will can evolve in physical theories that allow a continuous amount of free will to be exercised, that is from completely unbiased to completely biased choosing. Then, we will demonstrate that quantum physics permits free will, whereas classical physics does not.

To address the problem of free will and its neurophysiological support in the brain, we first introduce a precise quantitative measure of free will based on the classical bits of Shannon information gained when an agent makes a choice at points of bifurcation in its physical dynamics (Section 2). Next, we scrutinize the intractability of free will within classical physics and pinpoint its origin in the deterministic Hamilton's equations (Section 3). Then, we explain how modern quantum theory furnishes a physical measurement process in which conscious agents are able to exercise their free will (Section 4), and elaborate on the neurophysiological mechanisms that could evolve through natural selection to harness the possible biasing/unbiasing of inherent quantum propensities as provided by quantum indeterminism (Section 5). Further, we explore the implications of the presented quantum approach for personal responsibility, ethics, and moral values (Section 6). Lastly, we conclude with a discussion (Section 7) on synaptic learning through trial-and-error mechanism and elucidate how positive or negative feedback could affect the amount of free will possessed by synapses in the brain cortex.

\section{Quantitative measure of free will}

Free will is characterized by the capacity of conscious agents to make a genuine choice among several (at least two) alternative future courses of action. The ability to do otherwise is an essential ingredient of the act of choosing $[2-4,14-16]$. Furthermore, the choice needs to be exercised in the absence of external coercion in order to be free. Our internal desires, however, may influence and bias the probabilities with which different alternatives are actualized. For example, suppose that you are given the choice of having an ice cream with either vanilla or chocolate flavor (Figure 1). If the two choice outcomes are equiprobable due to equal desires, namely, both are $50 \%$, then the choice is unbiased and completely free. However, if 
one of the probabilities is larger, say $75 \%$ for vanilla and $25 \%$ for chocolate, then the choice is biased and only partially free. When one of the probabilities becomes absolutely certain, say $100 \%$ for vanilla and $0 \%$ for chocolate, then the choice is not free at all.

Before the actual choice of the agent is executed, each of the above three cases has a different probability distribution $P(X)$ with corresponding Shannon entropy $H(X)$ measured in bits $[17,18]$

$$
H(X)=-\sum_{k} P\left(x_{k}\right) \log _{2} P\left(x_{k}\right)
$$

where $X$ is the discrete random variable with possible outcomes $x_{1}, x_{2}, \ldots, x_{k}$ that occur with probabilities $P\left(x_{1}\right), P\left(x_{2}\right), \ldots, P\left(x_{k}\right)$. The function $f(P)=-P \log _{2} P$ is concave within the unit interval $P \in[0,1]$, where by convention $0=0 \log _{2} 0$ [19].

The information content (also called surprisal) of an individual outcome $x_{k}$ is defined as

$$
I\left(x_{k}\right)=-\log _{2} P\left(x_{k}\right) .
$$

Therefore, the Shannon entropy of a distribution $P(X)$ is the average (expected) information content of the outcome of a random trial

$$
H(X)=\sum_{k} P\left(x_{k}\right) I\left(x_{k}\right)
$$

A closely related concept, which considers the dynamic update from an initial probability distribution $P_{i}(X)$ to a final probability distribution $P_{f}(X)$, is the information gain $D\left(P_{f} \| P_{i}\right)$ (also called KullbackLeibler divergence or discrimination distance) given by [20, 21]

$$
D\left(P_{f} \| P_{i}\right)=\sum_{k} P_{f}\left(x_{k}\right) \log _{2}\left[\frac{P_{f}\left(x_{k}\right)}{P_{i}\left(x_{k}\right)}\right] .
$$

In the case when the final distribution is peaked onto a single outcome $x_{k}$, namely, $P_{f}\left(x_{k}\right)=1$, the information gain reduces to the information content (surprisal) (2) resulting from occurrence of the individual outcome $x_{k}$

$$
D\left(P_{f}\left(x_{k}\right)=1 \| P_{i}\right)=-\log _{2} P_{i}\left(x_{k}\right) .
$$

Consequently, weighted averaging over all possible single-outcome information gains with their corresponding probabilities of occurrence $P_{i}\left(x_{k}\right)$ returns the Shannon entropy of the initial probability distribution

$$
\sum_{k} P_{i}\left(x_{k}\right) D\left(P_{f}\left(x_{k}\right)=1 \| P_{i}\right)=H\left(P_{i}(X)\right) .
$$

Therefore, the Shannon entropy $H(X)$ of a distribution $P(X)$ is also the average (expected) information gain from learning the outcome of a random trial.

Consider now a conscious agent who is given the choice of having an ice cream, where he or she is able to select either vanilla or chocolate flavor. The expected information gain from learning the actual choice performed by the agent could be identified with the amount of free will $\mathcal{F}$ exercised by the agent. Thus, free will is quantifiable through the Shannon entropy $H_{i}$ of the initial probability distribution $P_{i}(X)$ determined by the physical laws that govern the time dynamics of the agent up to the point of bifurcation of future trajectories

$$
\mathcal{F}=-\sum_{k} P_{i}\left(x_{k}\right) \log _{2} P_{i}\left(x_{k}\right)
$$

The amount of free will $\mathcal{F}$ is measured in bits and represents the expected (average) surprisal resulting from the act of choosing (Figure 1). The main difference between (7) and (1) is that the Shannon entropy $H(X)$ could also be applied to quantify "fictitious" probability distributions representing personal ignorance about the actual physical state of the world $[22,23]$, whereas the amount of free will $\mathcal{F}$ is defined only with respect to the fundamental physical laws that govern the dynamics of the physical system. 


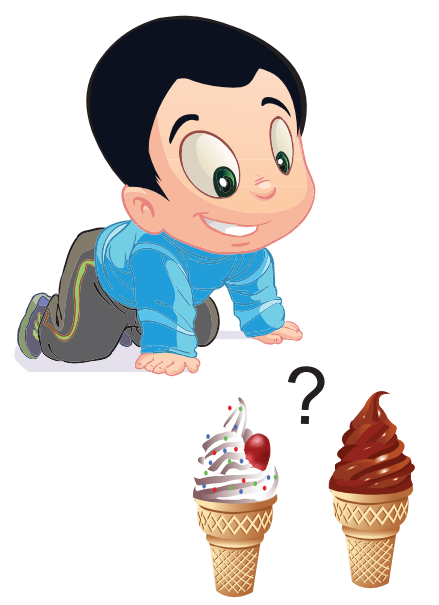

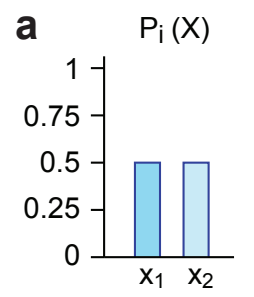

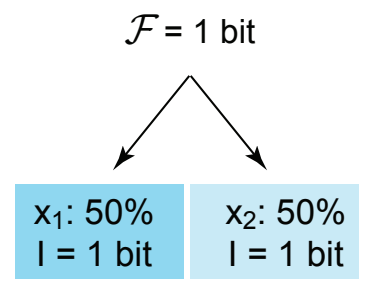

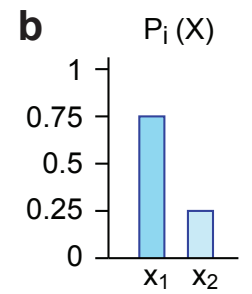

$\mathcal{F}=0.8$ bits

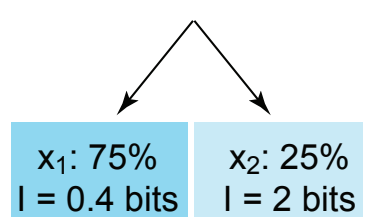

C

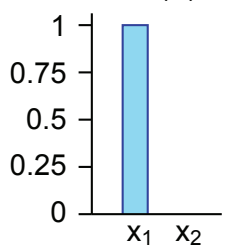

$\mathcal{F}=0$ bits

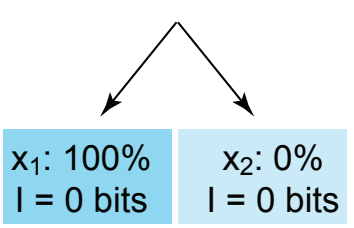

Figure 1: The amount of free will exercised by a conscious agent could be quantified by the expected (average) information gain from learning the actual choice performed by the agent. For illustration is used the simplest case of a probability distribution $P_{i}(X)$ with only two outcomes $x_{1}$ and $x_{2}$, where the corresponding probabilities are interpreted as inherent propensities. If the two initial probabilities are equiprobable, namely, both are $50 \%$, then each choice delivers 1 bit of new information indicating that the act of choosing was unbiased and completely free (a). However, if one of the initial probabilities is larger, $75 \%$ vs $25 \%$, then the average choice delivers only 0.8 bits of new information indicating that the act of choosing was biased and only partially free (b). When one of the initial probabilities is absolutely certain, $100 \%$ vs $0 \%$, then the choice delivers no new information indicating that the act of choosing was not free at all (c). Legend: $\mathcal{F}$ denotes the amount of free will, whereas $I$ denotes the information gain for each particular outcome.

In an ideal setting allowing for the same act of choosing to be repeated multiple times, $t_{1}, t_{2} \ldots, t_{n}$, the existing biases will be manifested in the limit of large number of repetitions $n$, where the relative frequency $n_{k} / n$ of the outcome $x_{k}$ (that occurred $n_{k}$ number of times) approaches the initial probability $P_{i}\left(x_{k}\right)$, namely

$$
\lim _{n \rightarrow \infty} \frac{n_{k}}{n}=P_{i}\left(x_{k}\right)
$$

The quantitative measure of free will $\mathcal{F}$ given by (7) is consistent with our intuitive expectation that the amount of free will should be independent on whether we make two choices simultaneously or not. Indeed, suppose that we have to make two consecutive unbiased choices for type of ice cream (vanilla or chocolate) and type of container (waffle cone or plastic cup). In such case, the resulting amount of free will exercised is 2 bits, which is the sum of two 1-bit choices. Alternatively, if we have to choose one of the four available combinations of ice cream and container at once, then we will exercise a single 2-bit choice. Thus, the overall amount of free will is the same as in the case with two consecutive choices.

\section{Intractability of free will in classical physics}

Determinism is the main characteristic feature of classical physics [1]. A physical theory is deterministic, if from the current state $S(t=0)$ of a closed physical system, the physical laws permit mathematical prediction of any future state $S(t>0)$ with absolute certainty and arbitrarily high precision. In other words, probabilities in deterministic theories do not have a fundamental physical origin, but can only reflect subjective ignorance with respect to the objective physical reality of the surrounding world.

Phase space description. In classical mechanics, the physical state of a system is mathematically represented by a point in phase space [9]. The phase space is a multi-dimensional abstract space in which every degree of freedom of the physical system is represented by an axis. For a composite system of $n$ particles, the phase space contains $3 n$ canonical position coordinates $q_{i}$, representing the $x, y, z$ position components of each particle, and $3 n$ canonical momentum coordinates $p_{i}$, representing the $p_{x}, p_{y}, p_{z}$ momentum components 
of each particle. The state of the system $S\left(q_{i}, p_{i}, t\right)$ evolves in time according to the system of Hamilton's equations

$$
\frac{d q_{i}}{d t}=\frac{\partial H\left(q_{i}, p_{i}, t\right)}{\partial p_{i}}, \quad \frac{d p_{i}}{d t}=-\frac{\partial H\left(q_{i}, p_{i}, t\right)}{\partial q_{i}},
$$

where $d$ is is total derivative operator, $\partial$ is partial derivative operator, and the Hamiltonian $H\left(q_{i}, p_{i}, t\right)$ is a distinguished physical observable corresponding to the total energy of the system.

Solving Hamilton's equations for a classically admissible Hamiltonian shows that the dynamic trajectories of classical physical systems are continuous paths in phase space that do not contain any genuine bifurcation points. This implies that free will is impossible in classical physics. The main culprit for this state of affairs is the incompatibility between free will and determinism. Indeed, if the state of the physical system is a point in phase space, the Shannon entropy of the initial probability distribution is always zero, $H\left(P_{i}(X)\right)=0$, and according to $(7)$ there is no information gain and no free will, $\mathcal{F}=0$.

The only way that probabilities can occur in classical physics is due to ignorance of the initial state $S(t=0)$ of the physical system. Suppose that our measurement instruments operate with some finite precision and we can locate with probability $P$ the initial state of measured physical system to a certain volume in phase space consisting of all positions between $q_{i}$ and $q_{i}+\Delta q_{i}$ and all momenta between $p_{i}$ and $p_{i}+\Delta p_{i}$. The probability density is $\rho=P / V$, where the volume in phase space is $V=\prod_{i} \Delta q_{i} \Delta p_{i}$. The resulting dynamic evolution from Hamilton's equations (9) obeys Liouville's theorem according to which the phase-space flow is like an incompressible fluid [24, 25]

$$
\frac{d \rho}{d t}=\frac{\partial \rho}{\partial t}+\sum_{i}\left(\frac{\partial \rho}{\partial q_{i}} \frac{d q_{i}}{d t}+\frac{\partial \rho}{\partial p_{i}} \frac{d p_{i}}{d t}\right)=0 .
$$

This implies that the probability density remains constant and the physical system occupies the same volume in phase space at all times. In other words, the number of microstates neither increases, nor decreases, which is a manifestation of the fact that dynamic trajectories neither bifurcate, nor merge. Whether we know or do not know the exact microstate of the classical physical system, has no bearing on the inability of classical systems to make choices.

Chaotic dynamics. Nonlinear dynamics in multipartite classical systems can lead to deterministic chaos [26]. Chaotic behavior is characterized with extreme sensitivity to small perturbations, manifestation of irregular orbits that explore the entire phase space, and separation of infinitesimally close orbits at an exponentially fast rate, which is quantifiable in terms of the Lyapunov exponent [27]. Taken together, these features of chaotic systems establish a short-term predictability time window during which the actual orbit of the system does not deviate significantly from the predicted orbit. For longer times, however, the dynamics of chaotic systems becomes practically unpredictable due to exponential amplification of tiny errors in the empirical measurement of the initial state of the system [28, 29]. This kind of effective unpredictability, which is due to our lack of knowledge, skills or technologies to predict the deterministic orbits far enough into the future [30], has no bearing on the fact that classical deterministic systems are unable to make choices [1]. In fact, a classical deterministic universe has no internal source of perturbations, which means that under the phrase "small perturbations" the classical physicist hides any errors that arise due to the simplifying assumption that the physical system of interest is closed and the rest of the universe can be ignored. Alternatively, working with a finite numerical precision while solving a system of differential equations [31] could be viewed as introducing fictitious perturbations at each computational step where numerical rounding is employed. Since free will is the inherent capacity of physical systems to make genuine choices, it cannot be rescued by mere consideration of instability and chaos in complex classical systems. Mathematical modeling of genuine choices requires the introduction of physical propensities and indeterminism.

Incompatibilism. The philosophical stance that free will is incompatible with physical determinism is referred to as incompatibilism. Deducing incompatibilism, however, is only the starting point of any serious investigation of the problem of free will. Before the discovery of quantum mechanics in 1920s, all leading 
physical theories, including Newtonian, Lagrangian, and Hamiltonian mechanics, as well as Maxwell's electrodynamics, were deterministic. This meant that any classical physical theory of consciousness was forced to accept determinism and abandon free will. Without free will there can be no moral responsibility, at least no more than a stone is morally responsible for breaking someone's leg or a car is morally responsible for not moving without petrol fuel [32]. Under ordinary circumstances, arriving at a contradiction with our own introspective testimony of free will (for a list of such contradictions see 7) would be viewed as an indication to reject the faulty premise, which happens to be classical physics. Yet, there are still some philosophers and/or neuroscientists who reject free will $[11,13,33]$ based on the outdated belief in determinism resulting from Hamilton's equations. Before 1920s, classical physics was essentially all of physics, which meant that to defend free will one had to abandon the physicalism altogether. Thus, it is not surprising that a number of prominent 19th century philosophers chose physicalism over free will $[10,34]$. With the advent of modern quantum mechanics, however, we now know that classical mechanics in inadequate to describe the physical world and physical reality is governed by indeterministic quantum physical laws. Modern quantum physics no longer clashes with the existence of free will and supports the possibility of genuine choice making.

\section{Quantum indeterminism and free will}

Having quantified the amount of free will $\mathcal{F}$, which is intimately linked to the fundamental physical laws that govern the dynamics of physical systems, we can use (7) as a tool to demonstrate that free will may evolve through natural selection in physical agents that inhabit a quantum indeterministic world. The important thing to note is that in an indeterministic world, the physical laws may predict different probability distributions for different situations. In the presence of genuine bifurcations of the dynamic trajectories for future courses of action, the choices of the physical agents may have important consequences about whether the agent will have the same abundance of future courses of action to choose from. For example, based on the outcome of a past choice some of the previously available trajectories may now obtain zero probability of future occurrence. To make these ideas more tangible, first we will briefly explain how the Schrödinger equation and the Born rule are used to predict the probabilities for different outcomes in quantum measurements. Then, we will illustrate how quantum theory supports the full range of actions, from completely unbiased to completely biased, using the famous Stern-Gerlach experiment implementing alternative (incompatible) quantum measurements of the spin components of a single qubit.

Schrödinger equation. The main quantum physical law that governs what exists and how it evolves in time is given by the Schrödinger equation [35, 36]

$$
\imath \hbar \frac{\partial}{\partial t}|\Psi(\mathbf{r}, t)\rangle=\hat{H}|\Psi(\mathbf{r}, t)\rangle
$$

where $\imath=\sqrt{-1}$ is the imaginary unit, $\hbar$ is the reduced Planck constant, $\frac{\partial}{\partial t}$ is the partial derivative operator with respect to time, $|\Psi(\mathbf{r}, t)\rangle$ is the quantum state vector, $\mathbf{r}=(x, y, z)$ is the vector of position coordinates, $t$ is time, and $\hat{H}$ is the Hamiltonian operator corresponding to the total energy of the quantum system $[1,37-39]$.

The quantum state vector $|\Psi(\mathbf{r}, t)\rangle$ of the physical system represents a continuous distribution of quantum probability amplitudes, which exhibit wave-like properties in 3-dimensional space [40, 41]. For example, the linearity of the Schrödinger equation implies that any two solutions $\left|\Psi_{1}(\mathbf{r}, t)\right\rangle$ and $\left|\Psi_{2}(\mathbf{r}, t)\right\rangle$ can interfere with each other in the form of a linear quantum superposition

$$
\left|\Psi_{s}(\mathbf{r}, t)\right\rangle=a_{1}\left|\Psi_{1}(\mathbf{r}, t)\right\rangle+a_{2}\left|\Psi_{2}(\mathbf{r}, t)\right\rangle,
$$

where $a_{1}$ and $a_{2}$ are complex coefficients. Due to the principle of quantum superposition, the quantum state behaves like a vector in an abstract Hilbert space. For an $n$-dimensional Hilbert space $\mathcal{H}$, the quantum state 
is an $n \times 1$ column vector called a ket [42-44]

$$
|\Psi(\mathbf{r}, t)\rangle=\left(\begin{array}{c}
a_{1} \\
a_{2} \\
\vdots \\
a_{n}
\end{array}\right) .
$$

The complex conjugate transpose of the ket is a $1 \times n$ row vector called a bra

$$
\langle\Psi(\mathbf{r}, t)|=\left(\begin{array}{llll}
a_{1}^{*} & a_{2}^{*} & \ldots & a_{n}^{*}
\end{array}\right)
$$

residing in a dual Hilbert space $\mathcal{H}^{*}$ [45]. Normalization of physical probabilities requires that the quantum states have a unit norm

$$
\langle\Psi(\mathbf{r}, t) \mid \Psi(\mathbf{r}, t)\rangle=\sum_{n} a_{n}^{*} a_{n}=\sum_{n}\left|a_{n}\right|^{2}=1 .
$$

Born rule. Quantum indeterminism is manifested in the act of quantum measurement, which is governed by the Born rule $[46,47]$. What can be measured as observable physical quantities are the eigenvalues $\lambda_{1}, \lambda_{2}, \ldots, \lambda_{n}$ of some quantum operator (also called quantum observable) $\hat{A}$, which is represented by an $n \times n$ matrix and indicated with the hat symbol [36, 43, 48, 49]. The quantum observable $\hat{A}$ may operate upon any input quantum state and return another output quantum state [50]. However, of special physical significance is the set of eigenvectors $\left|\Phi_{1}\right\rangle,\left|\Phi_{2}\right\rangle, \ldots,\left|\Phi_{n}\right\rangle$ of $\hat{A}$ such that the action of $\hat{A}$ on an eigenvector $\left|\Phi_{n}\right\rangle$ returns the same eigenvector $\left|\Phi_{n}\right\rangle$ multiplied by the corresponding eigenvalue $\lambda_{n}$, namely, $\hat{A}\left|\Phi_{n}\right\rangle=\lambda_{n}\left|\Phi_{n}\right\rangle$ [51]. The eigenvectors and eigenvalues of quantum observables allow a spectral decomposition in the form

$$
\hat{A}=\sum_{n} \lambda_{n}\left|\Phi_{n}\right\rangle\left\langle\Phi_{n}\right|=\left(\begin{array}{cccc}
\lambda_{1} & 0 & 0 & 0 \\
0 & \lambda_{2} & 0 & 0 \\
0 & 0 & \ddots & 0 \\
0 & 0 & 0 & \lambda_{n}
\end{array}\right),
$$

where $\left|\Phi_{1}\right\rangle\left\langle\Phi_{1}|,| \Phi_{2}\right\rangle\left\langle\Phi_{2}|, \ldots,| \Phi_{n}\right\rangle\left\langle\Phi_{n}\right|$ are the individual projection operators onto the rays $\left|\Phi_{1}\right\rangle,\left|\Phi_{2}\right\rangle, \ldots,\left|\Phi_{n}\right\rangle$ in the Hilbert space $\mathcal{H}$.

The Born rule states that if the quantum observable $\hat{A}$ is measured on a quantum physical system in state $|\Psi\rangle$, then the expected value $\bar{A}$ (determined as weighted average over all observable outcomes) is

$$
\bar{A}=\langle\Psi|\hat{A}| \Psi\rangle=\sum_{n} \lambda_{n}\left\langle\Psi \mid \Phi_{n}\right\rangle\left\langle\Phi_{n} \mid \Psi\right\rangle=\sum_{n} \lambda_{n} a_{n}^{*} a_{n}=\sum_{n}\left|a_{n}\right|^{2} \lambda_{n},
$$

where $a_{n}=\left\langle\Phi_{n} \mid \Psi\right\rangle$ is the projected quantum probability amplitude from the state $|\Psi\rangle$ onto the state $\left|\Phi_{n}\right\rangle$, $a_{n}^{*}$ is the complex conjugate of $a_{n}$, and $\left|a_{n}\right|^{2}$ is the quantum probability for the measuring device to register the observable outcome $\lambda_{n}$.

Alternative measurements of the quantum spin of a single qubit. Different quantum probability distributions arise for alternative (incompatible) quantum measurements as illustrated by the Stern-Gerlach experiment with silver atoms [52-54]. Each silver atom is a simple two-level quantum system (qubit) that exhibits only two possible values $\pm \frac{1}{2}$ (in $\hbar$ units) of the quantum spin observable $\hat{S}$.

The quantum spin of a qubit can point along an arbitrary $u$-axis inside the real 3-dimensional space. Expressed in spherical coordinates $(r, \theta, \varphi)$, with the normalization condition $r=1$ taken into account, the spin observable $\hat{S}_{u}$ along the $u$-axis can be written in terms of the Pauli spin matrices $\hat{\sigma}_{x}, \hat{\sigma}_{y}$ and $\hat{\sigma}_{z}$ as

$$
\hat{S}_{u}=\sin \theta \cos \varphi \hat{\sigma}_{x}+\sin \theta \sin \varphi \hat{\sigma}_{y}+\cos \theta \hat{\sigma}_{z}
$$


a

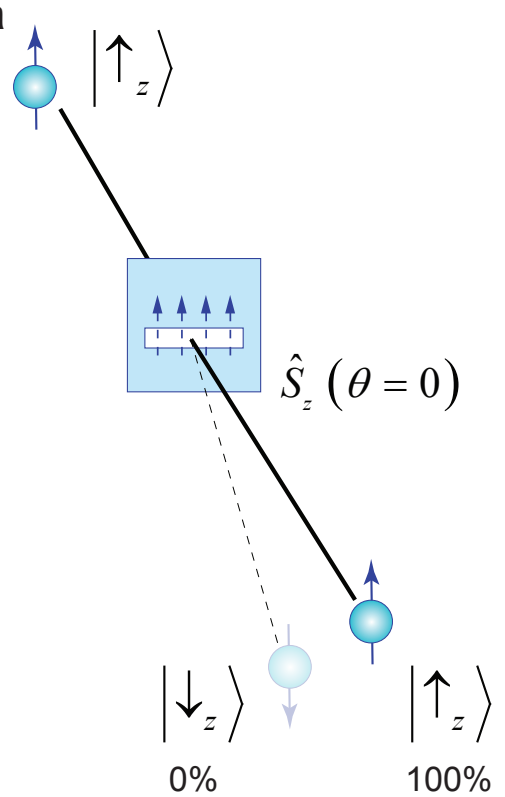

b

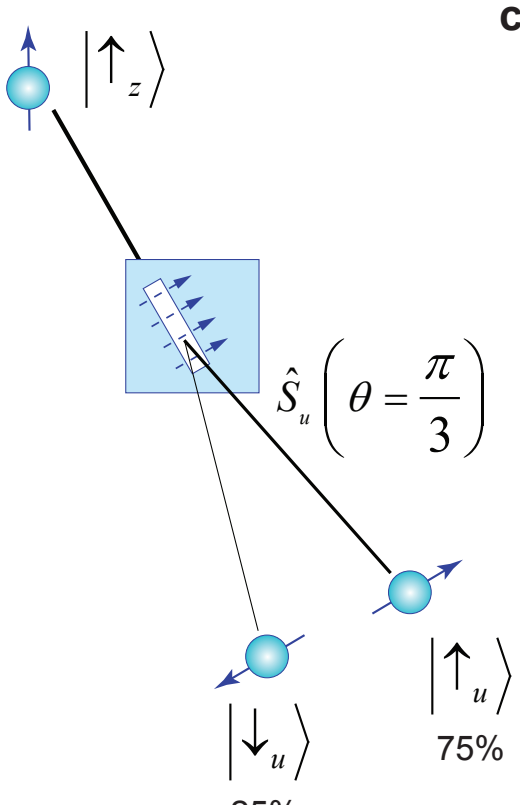

C

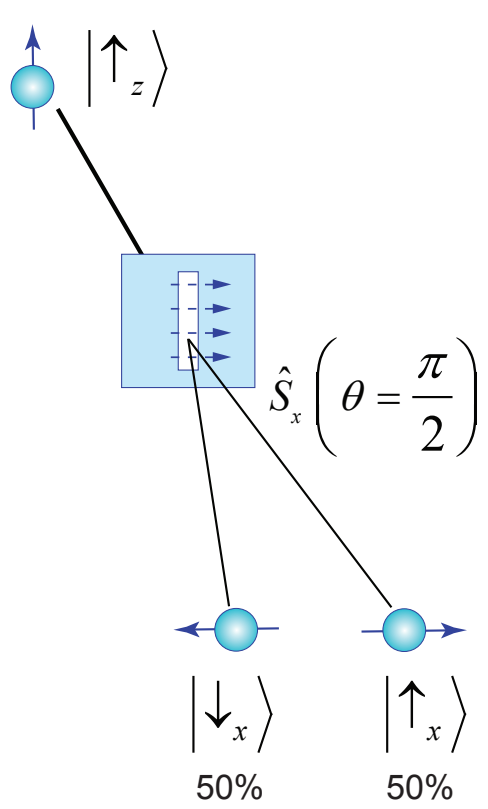

Figure 2: Stern-Gerlach experiment with a silver atom (qubit) whose initial state is $|\Psi\rangle=|\uparrow z\rangle$, which passes through inhomogeneous magnetic field rotated at a polar angle $\theta$. Depending on which quantum observable is measured, the outcomes can vary from completely deterministic to completely indeterministic: (a) If $S_{z}(\theta=0)$ is measured, the observable outcome $\left|\uparrow_{z}\right\rangle$ occurs with probability of $100 \%$ and $\left|\downarrow_{z}\right\rangle$ with probability of $0 \%$. (b) If $\hat{S}_{u}\left(\theta=\frac{\pi}{3}\right)$ is measured, the observable outcome $\left|\uparrow_{u}\right\rangle$ occurs with probability of $75 \%$ and $\left|\downarrow_{u}\right\rangle$ with probability of $25 \%$. (c) If $\hat{S}_{x}\left(\theta=\frac{\pi}{2}\right)$ is measured, the observable outcome $\left|\uparrow_{x}\right\rangle$ occurs with probability of $50 \%$ and $\left|\downarrow_{x}\right\rangle$ with probability of $50 \%$.

If we perform experiments with a qubit that is initially prepared in an eigenstate of the $\hat{S}_{z}$ observable, it will be convenient to express all quantum states and observables in the $\left|\uparrow_{z}\right\rangle,\left|\downarrow_{z}\right\rangle$ basis as follows

$$
\begin{gathered}
\left|\uparrow_{z}\right\rangle=\left(\begin{array}{c}
1 \\
0
\end{array}\right), \quad\left|\downarrow_{z}\right\rangle=\left(\begin{array}{c}
0 \\
1
\end{array}\right), \\
\hat{\sigma}_{x}=\left(\begin{array}{cc}
0 & 1 \\
1 & 0
\end{array}\right), \quad \hat{\sigma}_{y}=\left(\begin{array}{cc}
0 & -\imath \\
\imath & 0
\end{array}\right), \quad \hat{\sigma}_{z}=\left(\begin{array}{cc}
1 & 0 \\
0 & -1
\end{array}\right), \\
\hat{S}_{u}=\left(\begin{array}{cc}
\cos \theta & e^{-\imath \varphi} \sin \theta \\
e^{\imath \varphi} \sin \theta & -\cos \theta
\end{array}\right),
\end{gathered}
$$

where $\theta$ is the polar angle and $\varphi$ is the azimuthal angle of the $u$-axis. The eigenvectors of $\hat{S}_{u}$ are

$$
\begin{aligned}
& \left|\uparrow_{u}\right\rangle=\cos \left(\frac{\theta}{2}\right)\left|\uparrow_{z}\right\rangle+\sin \left(\frac{\theta}{2}\right) e^{\imath \varphi}\left|\downarrow_{z}\right\rangle, \\
& \left|\downarrow_{u}\right\rangle=-\sin \left(\frac{\theta}{2}\right)\left|\uparrow_{z}\right\rangle+\cos \left(\frac{\theta}{2}\right) e^{\imath \varphi}\left|\downarrow_{z}\right\rangle,
\end{aligned}
$$

with corresponding eigenvalues of $+\frac{1}{2}$ for $\left|\uparrow_{u}\right\rangle$ and $-\frac{1}{2}$ for $\left|\downarrow_{u}\right\rangle$.

Substitution of different values for the polar and azimuthal angles gives the eigenvectors for the Pauli matrices: the eigenvectors $\left|\uparrow_{x}\right\rangle,\left|\downarrow_{x}\right\rangle$ of $\hat{S}_{x}=\hat{\sigma}_{x}$ are obtained for $\theta=\frac{\pi}{2}$ and $\varphi=0$, the eigenvectors $\left|\uparrow_{y}\right\rangle,\left|\downarrow_{y}\right\rangle$ of $\hat{S}_{y}=\hat{\sigma}_{y}$ are obtained for $\theta=\frac{\pi}{2}$ and $\varphi=\frac{\pi}{2}$, and the eigenvectors $\left|\uparrow_{z}\right\rangle,\left|\downarrow_{z}\right\rangle$ of $\hat{S}_{z}=\hat{\sigma}_{z}$ are obtained for $\theta=0$ and $\varphi=0$. 
Now we are ready to compute the quantum probability distributions for a qubit whose initial state is $|\Psi\rangle=$ $\left|\uparrow_{z}\right\rangle$, which passes through a Stern-Gerlach apparatus measuring one of three alternative (incompatible) spin observables: $\hat{S}_{z}(\theta=0), \hat{S}_{u}\left(\theta=\frac{\pi}{3}\right)$ or $\hat{S}_{x}\left(\theta=\frac{\pi}{2}\right)$, where without loss of generality we have set $\varphi=0$. In the case when $\hat{S}_{z}$ is measured (Figure $2 \mathrm{a}$ ), the observable outcome $\left|\uparrow_{z}\right\rangle$ occurs with probability of $100 \%$ and $\left|\downarrow_{z}\right\rangle$ with probability of $0 \%$. In other words, because the initial state $|\Psi\rangle=\left|\uparrow_{z}\right\rangle$ is an eigenvector of the measured observable $\hat{S}_{z}$, the outcome is absolutely certain and deterministic. In fact, the capacity to produce a deterministic outcome upon measurement of a quantum observable is a physical way of defining what an eigenvector of that quantum observable is. In the case when $\hat{S}_{u}\left(\theta=\frac{\pi}{3}\right)$ is measured (Figure $2 \mathrm{~b}$ ), the observable outcome $\left|\uparrow_{u}\right\rangle$ occurs with probability of $75 \%$ and $\left|\downarrow_{u}\right\rangle$ with probability of $25 \%$. The observable outcomes are indeterministic but biased. Completely unbiased indeterministic outcomes are obtained when $\hat{S}_{x}$ is measured (Figure 2c), namely, the observable outcome $\left|\uparrow_{x}\right\rangle$ occurs with probability of $50 \%$ and $\left|\downarrow_{x}\right\rangle$ with probability of $50 \%$. Taken together, these alternative experimental conditions illustrate a crucial fact, namely, the act of quantum measurement supports the whole range of probability distributions, from completely deterministic to completely indeterministic, depending on the measured physical observable parametrized by $\theta$. In other words, stating that quantum physics is indeterministic should be understood in the sense that quantum physics admits indeterminism as a possibility without excluding determinism as a special case of biased quantum probability distribution.

Noteworthy, quantum probabilities remain invariant with respect to interchange of the initial and final states during quantum measurement. For example, consider an initial state $|\Psi\rangle$ and final state $\left|\Phi_{n}\right\rangle$. The probability $P_{n}$ to observe the transition $|\Psi\rangle \rightarrow\left|\Phi_{n}\right\rangle$ in the act of quantum measurement is given by the expectation value of the projection operator $\left|\Phi_{n}\right\rangle\left\langle\Phi_{n}\right|$ for the initial state $|\Psi\rangle$ according to the Born rule

$$
P_{n}=\left\langle\Psi \mid \Phi_{n}\right\rangle\left\langle\Phi_{n} \mid \Psi\right\rangle=a_{n}^{*} a_{n}=\left|a_{n}\right|^{2} .
$$

Because the multiplication of complex quantum probability amplitudes is commutative (namely, the order of multiplication does not matter, $\left.a_{n}^{*} a_{n}=a_{n} a_{n}^{*}\right), P_{n}$ is also the probability to observe the converse transition $\left|\Phi_{n}\right\rangle \rightarrow|\Psi\rangle$ given by the expectation value of the projection operator $|\Psi\rangle\langle\Psi|$ for the initial state $\left|\Phi_{n}\right\rangle$ as follows

$$
P_{n}=\left\langle\Phi_{n} \mid \Psi\right\rangle\left\langle\Psi \mid \Phi_{n}\right\rangle=a_{n} a_{n}^{*}=\left|a_{n}\right|^{2} .
$$

Thus, we have a second physical way to obtain alternative quantum probability distributions, namely, we can measure the same quantum observable $\hat{S}_{z}$ for different initial quantum states $\left|\uparrow_{z}\right\rangle,\left|\uparrow_{u}\right\rangle$ or $\left|\uparrow_{x}\right\rangle$. We already know that if $\left|\uparrow_{z}\right\rangle$ is the initial state, the observable outcome $\left|\uparrow_{z}\right\rangle$ occurs with probability of $100 \%$ and $\left|\downarrow_{z}\right\rangle$ with probability of $0 \%$. In the case when $\left|\uparrow_{u}\right\rangle$ is the initial state, the observable outcome $\left|\uparrow_{z}\right\rangle$ occurs with probability of $75 \%$ and $\left|\downarrow_{z}\right\rangle$ with probability of $25 \%$. Completely unbiased quantum probability distribution is obtained when $\left|\uparrow_{x}\right\rangle$ is the initial state, where the observable outcome $\left|\uparrow_{z}\right\rangle$ occurs with probability of $50 \%$ and $\left|\downarrow_{z}\right\rangle$ with probability of $50 \%$.

Equipped with the precise understanding of the Born rule (17), and knowing the two main physical ways for production of biased probability distributions in the process of quantum measurement, we are now ready to tackle the problem of how free will is able to evolve in biological systems by varying the parameter $\theta$.

\section{Neurophysiological mechanisms of free will}

Free will is often viewed as a binary property: the capacity of a conscious agent to do otherwise is either true or false by virtue of the physical laws. For centuries philosophers felt no urgent need to quantify free will in the presence of biased choices, because the verdict in classical physics was clear, namely, physical determinism forbids free will and there is no point in quantifying something that is an illusion. In the modern age of advanced quantum technologies, however, we know that the physical reality is nonclassical and quantum indeterminism endows quantum physical systems with inherent propensity to make genuine choices, thereby manifesting varying amount of free will depending on how biased those choices are.

Without the existence of genuinely quantum physical substrates in the brain, the conscious agents would not have been capable of harnessing quantum effects to support intelligence, thinking and decision making 
$[1,55-57]$. The interaction between the constituent quantum particles of the nervous system of an organism and the surrounding environment constitutes a quantum measurement in the act of which different observable outcomes could occur with different probabilities. Similarly to the Stern-Gerlach experiment discussed above, the environment would be able to measure alternative (incompatible) quantum observables of neurons, which would comprise a set of functional conformations of neural biomolecules with catalytic activities. For example, quantum chemistry research supports dynamic quantum effects such as quantum tunneling in the gating of voltage-gated ion channels [58-61] or in the zipping of SNARE proteins during neurotransmitter release $[38,62]$.

Deterministic synaptic neurotransmission in sensory or motor pathways. Electric spikes (action potentials) propagating along the neuronal projections inside the neural network are the main carriers of physical information and consume about half of the energy utilized for supporting clinical consciousness by the brain cortex [63]. Sensory information is encoded in the form of electric spikes by the peripheral sensory organs after which it is reliably transmitted across the synapses of the sensory pathways through the thalamus toward the brain cortex where it is consciously experienced [64-67]. Somatomotor information from the motor cortex is outputted again in the form of electric signals that are reliably transmitted across the synapses of the somatomotor pathways through the anterior horn of the spinal cord toward the muscles [68]. To achieve reliable signal transmission, the chemical synapses in these pathways operate in a deterministic fashion employing multivesicular release upon depolarization of the presynaptic axonal boutons [69, 70]. The exocytotic release of neurotransmitter molecules from multiple synaptic vesicles then generates large postsynaptic currents in the target neuron [69]. The reliable deterministic transmission of information between the brain cortex and the body (Figure 3) is of paramount importance for the survival of the organism through the execution of fight-or-flight responses.

Synaptic vesicle release is a physical process that can be represented by a particular quantum observable $\hat{A}=1|1\rangle\langle 1|+0| 0\rangle\langle 0|$, which can be spectrally decomposed into two coarse-grained eigenvectors denoted as $|1\rangle$ with eigenvalue 1 for release of at least one synaptic vesicle and $|0\rangle$ with eigenvalue 0 for no synaptic vesicles released [71]. Formally, the mathematical structure of $\hat{A}$ is the same as the spin observable projection operator $\left|\uparrow_{z}\right\rangle\left\langle\uparrow_{z}\right|$. Under normal physiological conditions, the probability of release of at least one synaptic vesicle in extracortical synapses in the sensory/somatomotor pathways is $100 \%$. This complete determinism implies that the quantum state of the extracortical synapse is $|\Psi\rangle=|1\rangle$ when the quantum measurement is performed, $\langle\Psi|\hat{A}| \Psi\rangle=1$, hence no free will is manifested.

Indeterministic synaptic neurotransmission in the brain cortex. Individual synapses of pyramidal neurons inside the neocortex and the hippocampus operate in an indeterministic fashion releasing either a single synaptic vesicle or none [71-74], which is consistent with possible direct involvement in the neural mechanisms supporting human consciousness and free will. Each cortical synapse (Figure 4) appears to possess only one functional release site at a given time [75] such that the probability for exocytosis is $0.35 \pm 0.23$ per axonal spike [76]. Membrane-bound SNARE protein complexes zip into four- $\alpha$-helix bundles that drive exocytosis by merging the synaptic vesicles with the presynaptic plasma membrane [77, 78]. Quantum vibrational excitons propagating along the protein $\alpha$-helices might be instrumental in producing indeterministic physical outcomes with the use of quantum tunneling through massive barriers that are imposed by external protein clamps [38, 79-82]. In the absence of electric spikes, each SNARE protein complex is clamped by the $\mathrm{Ca}^{2+}$ sensor protein synaptotagmin-1 to prevent spontaneous release from resting neurons [83-85]. Only when the presynaptic buttons are depolarized, the activation of voltage-gated calcium channels leads to entry of $\mathrm{Ca}^{2+}$ ions that assist in releasing the synaptotagmin-1 clamping action, enhancing the probability of quantum tunneling of the vibrational exciton through the barrier and zipping the SNARE protein complex [62]. Cortical pyramidal neurons form on average 7500 synapses onto target neurons [86], where each electrically excited axonal bouton of a cortical pyramidal neuron is almost twice more likely to fail than succeed in releasing neurotransmitter [62,63]. This means that the quantum state of the extracortical synapse is $|\Psi\rangle=\sqrt{0.35}|1\rangle+\sqrt{0.65}|0\rangle$ when the quantum measurement is performed, $\langle\Psi|\hat{A}| \Psi\rangle=0.35$, hence the amount of free will manifested through synaptic vesicle release is $\approx 0.934$ bits per synapse or $\approx 7005$ bits per cortical pyramidal neuron. The human brain cortex contains $1.634 \times 10^{10}$ neurons [87] of which at 

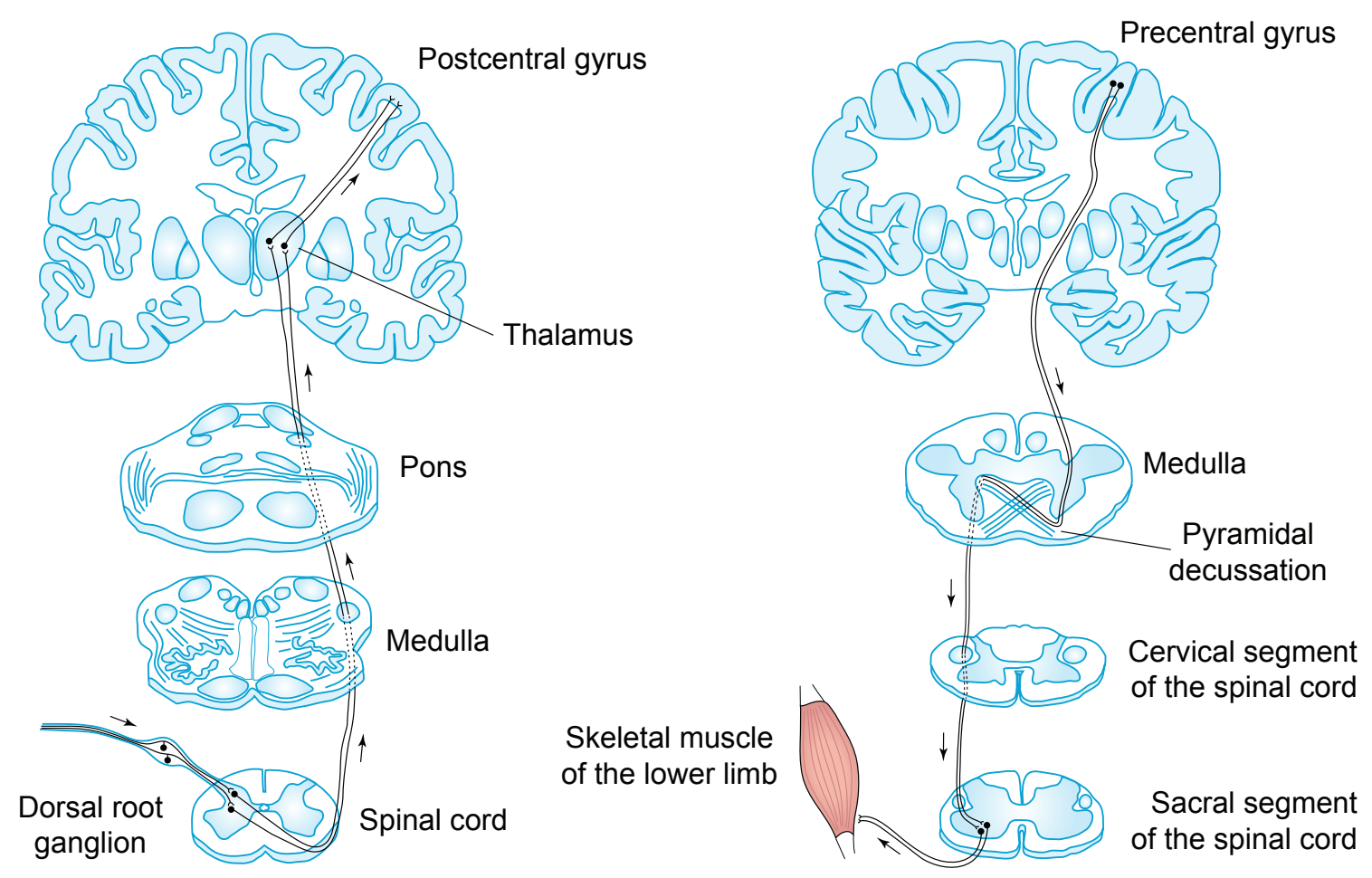

Figure 3: Classical communication through electric signals between the brain cortex and the body. The somatosensory pathway (left) delivers sensory information from the body to the somatosensory cortex in the postcentral gyrus, whereas the somatomotor pathway (right) delivers motor information from the motor cortex in the precentral gyrus to the body muscles. Deterministic release of synaptic vesicles lacks free will but ensures error-free perception of the surrounding world and guarantees reliable execution of fight-or-flight behavioral responses. The spinal cord segments, medulla and pons are represented with their transversal sections, whereas thalamus and cortex are shown in frontal slice. Modified from [1].

least $70 \%$ are excitatory pyramidal neurons [88]. If all cortical pyramidal neurons fire once, the amount of free will exercised would be over 80 terabits $\left(8 \times 10^{13} \mathrm{bits}\right)$. In other words, the expected information gain from learning which synapses are active and which synapses remain silent during an electric firing of all cortical pyramidal neurons is sufficient to exhaust the memory of a modern 10 terabyte hard drive (1 byte $=8$ bits). The energy power of $4.427 \mathrm{~W}$ consumed by the human brain cortex suffices to sustain an average firing frequency of $9.6 \mathrm{~Hz}$ [63]. Thus, the overall amount of free will provided by cortical synaptic activity is over 96 terabytes per second.

Taking stock of matters so far, we see that animal evolution through natural selection could have indeed optimized the amount of free will manifested by cortical or extracortical synapses in the nervous system. In order to receive reliable sensory information, the animal sensory organs and the sensory pathways need to operate deterministically with zero free will. Otherwise, the animal will receive various artifacts (errors) in the sensory picture of the world, which will result in inappropriate responses, possible injuries and ultimately death. Similarly, the somatomotor information outputted towards the muscles needs to be reliably transmitted for the execution of appropriate fight-or-flight responses.

Importance of free will in the evolution of prey-predator relationship. Consider a wild gazelle hunted by a pride of lions in the savanna. In order to outrun the predators, the motor cortex of the gazelle needs to control reliably the contraction of skeletal muscles, which also requires deterministic operation and lack of free will along the synapses of the somatomotor pathway. Failure of the gazelle to execute flawlessly the flight response [89] would lead to demise. The perfect communication between the brain cortex and the body, however, would not be very helpful for the prey animal if the overall behavior were deterministic and 


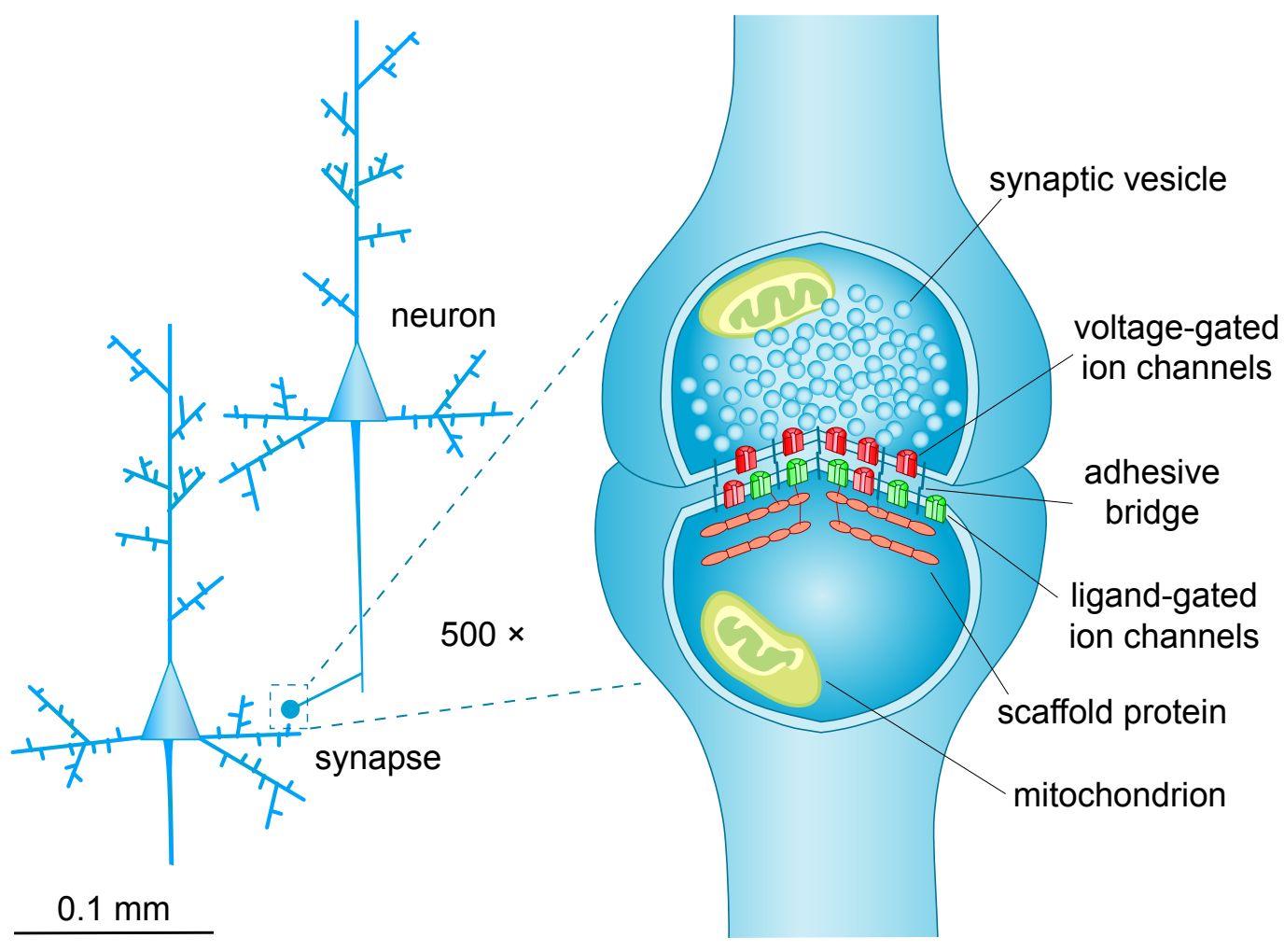

Figure 4: Excitatory synaptic contact between pyramidal cortical neurons. The presynaptic axonal bouton has a pool of synaptic vesicles that contain neurotransmitter. During an electric spike, the activation of presynaptic voltage-gated calcium channels initiates $\mathrm{Ca}^{2+}$ influx at the active zone, which may trigger fusion of a single synaptic vesicle with the plasma membrane. Varying probability of release provides different amounts of free will exercised in the synapses of the brain cortex. This inherent indeterminism supports higher cognitive decisions that are less predictable by adversaries and may enhance the survival of organisms. Following the successful exocytosis of a synaptic vesicle, the released neurotransmitter acts on postsynaptic receptors to induce postsynaptic electric currents in the target neuron. Structural support for the synapse is provided by adhesive bridges and scaffold proteins, whereas mitochondria ensure robust energy supply for synaptic neurotransmission. Modified from [1].

easily predictable by the predators. The hunting pride of lions usually attempts to ambush and encircle the prey. If the gazelle were running in a straight direction, its future path would be easily predictable by the lions and they would easily intercept it. That is why a certain amount of indeterministic performance of the brain cortex becomes an invaluable asset that can be further evolved to an optimal level through natural selection. Using its inherent free will, the gazelle is capable to unpredictably jump to the left or to the right, thereby confusing the lions and escaping through gaps in the circle left unattended by the lions. This is how enhancing the amount of free will possessed by the brain cortex may affect the animal behavior and have a survival value for the organism.

Importance of free will for human creativity. Divergent thinking characterizes creative thought during which are combined concepts and ideas that were previously thought to be unrelated [90-92]. Free will provides an inherent probabilistic mechanism for ideation of possible creative solutions, which are then critically assessed and their merits evaluated in a convergent process of elimination [93]. Successful creative solutions and human discoveries can then be transmitted in the form of art or passed down culturally from generation to generation $[94,95]$. 


\section{Free will and moral responsibility}

Free will is intimately connected with ethics and moral responsibility [96-99]. Conscious agents with free will are able to choose whether to do an action or not. In either way, action or inaction, the agents are morally responsible for the consequences of their choices, including any opportunities missed from the actions that they have chosen not to do. Moral responsibility, however, is contingent on the freedom of choice and the lack of external coercion. The amount of external coercion $\mathcal{C}$ could be quantified using the Kullback-Leibler divergence (4) from an initial distribution $P_{i}\left(x_{k}\right)$ characterizing the inherent desires of the agent to a final distribution $P_{f}\left(x_{k}\right)$ characterizing the available choices in the presence of coercive force

$$
\mathcal{C}=\sum_{k} P_{f}\left(x_{k}\right) \log _{2}\left[\frac{P_{f}\left(x_{k}\right)}{P_{i}\left(x_{k}\right)}\right] .
$$

In the case when a single course of action $x_{k}$ is forced onto an agent, $P_{f}\left(x_{k}\right)=1$, the amount of coercion reduces to the surprisal (2) resulting from occurrence of the individual outcome $x_{k}$ in the absence of coercion

$$
\mathcal{C}=-\log _{2} P_{i}\left(x_{k}\right) .
$$

In other words, there is no coercion, $\mathcal{C}=0$, if the agent would have chosen the outcome $x_{k}$ anyway, $P_{i}\left(x_{k}\right)=1$. The coercion is $\mathcal{C}=n$ bits for $P_{i}\left(x_{k}\right)=\left(\frac{1}{2}\right)^{n}$. In the limiting case when the agent has no desire to choose the outcome $x_{k}$ at all, namely, $P_{i}\left(x_{k}\right)=0$, the coercion becomes infinitely large, $\mathcal{C}=\infty$.

In classical deterministic physics, free will is impossible and the concept of coercion becomes meaningless. If the universe is a clockwork mechanism and humans are nothing but string puppets reacting to their physical circumstances, then nobody is accountable for anything. In the first half of 20th century, the famous trial lawyer Clarence Darrow successfully defended murderers from receiving the death penalty using the domino theory of moral nonresponsibility, namely, if someone's actions are always consequences of what others have done to him or her, then no one is responsible for anything he or she does [100, 101]. Indeed, we do not blame or punish falling stones for breaking someone's leg or static cars for not moving without petrol fuel [32].

In a quantum indeterministic world, however, free will is a valuable asset because it allows everyone to be able to choose his or her own purpose and meaning of life [1]. The free choices do not imply physical lawlessness. Instead, fundamental quantum physical laws including the Schrödinger equation and the Born rule determine the available future alternatives from which we are allowed to choose and how biased the probabilities for those different choices are. Because the acts of choosing are executed during quantum measurements performed by the surrounding environment upon the quantum state of the neural network, the organism is able to form memories of past choices and adapt the internal quantum dynamics so that the quantum probabilities for release of a synaptic vesicle per action potential can vary widely between cortical axonal buttons [76, 102-106]. Adjustment of synaptic vesicle release probabilities could be easily achieved by varying the potential energy barriers for quantum tunneling of vibrational excitons propagating along the $\alpha$-helices of SNARE proteins $[1,38,62,80]$. Changing the quantum probability distributions for synaptic release modifies the amount of free will manifested by a conscious agent at different stages in life.

Drug addiction, free will and moral responsibility. Narcotic drugs are able to elicit an initial surge of pleasurable sensation followed by a compulsive drive for self-administration of the drug $[107,108]$ mostly to avoid undesirable side effects of drug withdrawal [109] such as restlessness, irritability, insomnia, muscle and bone pain, muscle spasms, kicking movements of the legs, abdominal pain, diarrhea, vomiting, chills or cold flashes with goose bumps. If a drug addict is going to self-administer the drug with absolute certainty, then it would appear that there is no free will manifested. This, however, does not imply that the drug addict bears no moral responsibility for his or her actions, because one could rewind back the time until the first exposure to the addictive drug. At this point in time, if the subject was able to do otherwise but did not choose to do so (e.g., due to curiosity of trying what is it like to experience the surge of pleasurable sensation elicited by the narcotic drug), then the subject is fully responsible for his actions as he or she could have exercised at least $\mathcal{F}=1$ bit of free will. Alternatively, if the subject had no intention to try any drugs but 
the first drug administration was forced by someone else (e.g., drink spiking without subject's knowledge or permission, criminal intravenous injection, etc.), then the subject is not responsible for becoming an addict as he or she was subjected to an infinitely large coercion, $\mathcal{C}=\infty$. This clearly shows that because the amount of free will may dynamically change in time, attribution of moral responsibility and guilt should always take into account the entire history of previous choices made by the conscious subject.

Quantifying external coercion might be practically impossible for the assessment of putative effects of complex environmental factors. For example, a broker trading on the stock market may be influenced by a variety of uncontrollable external stochastic factors to exhibit so complicated behavior that it is unfeasible to deduce what could have had happened in the absence of some controllable factor. The measure given by (26), however, might be very useful for quantifying the effects of certain drugs upon the performance of humans engaged in some highly responsible professional activities that require execution of free choices. For example, any change from an initially unbiased probability of release $(p=0.5)$ will decrease the amount of free will exercised and will exert some non-zero coercion per synapse. Volatile anesthetics severely decrease the probability of synaptic vesicle release $(p \rightarrow 0)$ thereby erasing consciousness and free will [62]. Alternatively, narcotic drugs such as cocaine may increase the probability of synaptic vesicle release $(p \rightarrow 1)[110,111]$ thereby also reducing free will. Noteworthy, substances such as alcohol that induce failure of deterministic neurotransmission along the somatomotor pathways could be viewed as infinitely coercive, and are therefore appropriately banned for consumption by drivers of motor vehicles.

\section{Discussion}

Philosophers have produced volumes of literature debating free will in a binary fashion: a conscious agent either has free will or not. Determinism of classical physics bans the existence of agents with free will, which explains why the pursuit of compatibilism led to redefining of "free will" as something else that has nothing to do with the capacity of conscious agents to make genuine choices. Resorting to redefinition of "free will", however, does not solve the original problem and only helps create further confusion. Furthermore, in a deterministic physical world there can be no meaningful discussion of inherent biases or external coercion. Indeed, Hamilton's equations (9) imply that the dynamics of an agent is predetermined with absolute certainty, hence always completely biased and without free will. The latter fact precludes any attribution of responsibility or guilt to physical agents and serves as a foundation for erecting the domino theory of moral nonresponsibility. In classical physics, human ethics becomes a mere historic accident, as it is impossible to explain in what sense some moral value is objective. Modern quantum physics, however, provides fundamentally indeterministic physical laws that can naturally accommodate free will. When quantum physical systems are measured, they have the capacity to choose a single measurement outcome selected from a characteristic quantum probability distribution obeying the Born rule. The average information gain from learning the chosen measurement outcome, then serves as a quantitative indicator of the amount of free will possessed by the quantum system.

The precise quantification of the amount of free will allowed by quantum theory also illuminates old philosophical debates on the apparent tension between our capacity to do otherwise and our desire to rationally control our actions. If the physical laws were such that always either maximal free will is granted or no free will is granted at all, then we would not have had the opportunity to put ourselves in both of these situations depending on the context. Quantum theory resolves the tension by admitting varying amounts of free will for different measurement contexts. The neurobiology of different free will contexts is as follows: When the fundamental physical laws do not favor an outcome of our actions, there is a quantum physical observable in the neural system whose measurement outcomes are completely unbiased. For example, when we are born we know nothing about the external world and there could be a synapse whose probability of release of neurotransmitter is $50 \%$ for Yes and $50 \%$ for No. Without any inherent biases, we are maximally free to choose to do one thing or the other. Suppose that we choose Yes and the synapse releases neurotransmitter. If this decision leads to benefits, the molecular machinery for the neural dopamine reward system will be activated and the synapse may boost its probability for release to say $75 \%$. Conversely, if the decision leads to harmful consequences, the neural reward system will not be activated 
and the synapse may decrease its probability for release to say $25 \%$. Thus, we can learn from our previous successes or mistakes, trying to repeat the successes but not to repeat the mistakes again. The effect of learning new information upon an unbiased synapse will be to restrict the amount of manifested free will. Indeed, we feel comfortable when we live in a risk free environment. When everything goes smoothly in life, we do not want to choose differently and do not need any free will. This may explain the psychology behind denial of free will by accomplished philosophers. When things become stressful in life, however, we urgently need our free will and the capacity to choose otherwise. Subjected to high levels of adrenaline, synapses in the brain cortex that predictably activate or predictably remain silent, may be reset by experienced hardship in life to the unbiased state $50 \%$ for Yes and $50 \%$ for No. Thus, the presence or lack of relevant information could significantly impact the amount of free will that we exercise with respect to open questions for future action. The effect of learning upon free will could be in either direction: to decrease the amount of free will when we perform successfully or to enhance the amount of free will when we experience hardship in life. The suggested effect of learning upon the amount of synaptic free will is consistent with neurophysiological experiments, which have already shown that neuronal electric activity or inactivity is able to affect the probability of release of synaptic vesicles at individual synapses $[112,113]$.

Classical philosophers such as Arthur Schopenhauer, who lived and worked before the discovery of modern quantum physics in 1920s, viewed determinism as an inescapable obstacle to genuine free will. Consequently, they tried to redefine "free will" to indicate only willed or desired actions, which may not have been free. This only confuses matters because, although our emotions and desires influence our decisions, sometimes we exercise our free will to choose things that we do not desire. Also we do not have a persistent delusion that all bodily actions are caused by us. For example, if someone strikes the patellar ligament with a reflex hammer just below the knee cap, this will invariably cause our leg to kick out, but the accompanying conscious experience is as if the leg moved on its own without our conscious intention to move it [1]. Our subjective feeling of not using our free will to move the leg is indeed consistent with neuroanatomy: the patellar reflex is executed at the level of the spinal cord and the electric signal arrives at the brain cortex only after the leg motion has been already triggered. Thus, while the explanation of the patellar reflex is wanting in the absence of free will, it is quite simple if the existence of free will is acknowledged.

The presented quantum neurophysiology of free will vindicates the trustworthiness of our introspective testimonies of which actions we have freely chosen to do and which we have not. Because all newborns lack any knowledge about the surrounding world, they exercise their free will to explore various actions and experience their consequences. Here is where the importance of the dopamine reward system and the occurrence of pleasant or unpleasant feelings helps us learn through trial-and-error mechanism. The free will that we are endowed with allows us to choose what to become through our actions. We are born ignorant into this world, but our lives are too short to let everyone learn from his or her own mistakes. As a social species, we have contemplated on the latter fact and reached to the conclusion that while the ignorance of an adult is not an excuse for not bearing moral responsibility, we can excuse children by transferring temporarily the responsibility from the child to the parent who is engaged with the education of the child up to a certain age. Admitting the existence of free will and contemplating on social contexts in which the moral responsibility could be shared by a more experienced member of the social group is the starting point towards building an ethical system of moral values. In the animal kingdom, there are a number of extraordinary examples in which grandparents become responsible for the education of their grandoffspring, including whales, dolphins, elephants, monkeys and humans [114-124]. Of all animals, however, only humans teach their grandchildren abstract ideas (such as free will) and leave as a legacy a set of moral values. We hope that by raising the awareness of neuroscientists for the utility of modern quantum physics, the free will problem will no longer be intractable. The proposed quantitative measures of the amount of free will $\mathcal{F}$ or the presence of external coercion $\mathcal{C}$ might be useful for the development of legal policies to control drug use or for the attribution of guilt in legal cases.

\section{Conflict of Interest}

The author certifies that he has no affiliations with or involvement in any organization or entity with any financial interest, or non-financial interest in the subject matter or materials discussed in this manuscript. 


\section{Appendix: Problems in the philosophy of free will}

Quantum theory resolves many problems related to free will that were intractable in classical physics.

Locke's man in a locked room. John Locke argued that our belief in free will could be an illusion sustained by our ignorance. As an example, he considered a sleeping man who is transferred while asleep into a locked room where a desirable companionship is presented. When the man wakes up, he might choose to stay in the room by his own desire, without being aware that the room is locked and there is no way out. In this case, the man apparently stays in the room without having the option to have done otherwise. Yet, because the man's own desire has led him to choose the only available course of action, his ignorance of the real situation will sustain the illusion of free will [34]. Although superficially plausible, Locke's argument is based on a conditional statement and does not withstand further scrutiny. In fact, a real person will sooner or later decide to exit the room, which means that the illusion can persist only for a very limited period of time. In real life, we never experience a desire to move one of our arms or legs at a moment in time when the physical laws forbid such motion. This could fit under the premisses of classical physics only if our desires are always aligned with what is physically mandated by determinism thereby producing the illusion of free will. However, it is easy to find real life situations in which we freely choose a course of action reluctantly without any desire. For example, in a burning building a person may have to choose between going through the fire sustaining severe skin burns or jumping through high floor window sustaining severe bone fractures. Neither of these two choices is in any sense desirable and will never be experienced as desirable by the one who has to make them. Thus, since free will allows us to choose undesirable courses of action in real life, it is implausible that a physical mechanism can support the illusion of free will in the absence of free will.

Frankfurt's advanced device. Harry Frankfurt proposed a modern experiment aimed at establishing compatibilism between free will and determinism. He imagined an advanced device that is capable to continuously monitor and, if needed, trigger electric activity in a subject's brain. With such a device, it would be possible to do nothing if the subject chooses to do some action $A$, or trigger the device to electrically stimulate the execution of action $A$ if the subject has not chosen to do the action $A$. Similarly to Locke's argument, Frankfurt concludes that the subject can choose to do the action $A$, even though the action $A$ cannot be avoided [125]. The faulty reasoning is again in the conditional character of the statement. If the experiment is repeated several times, sooner or later the subject will chose not to do the action $A$ and be surprised that the action $A$ is executed anyway. Thus, compatibilism cannot be true. Availability of alternate possibilities is essential for the existence of free will.

Epiphenomenal belief in free will cannot evolve through natural selection. Psychological experiments have found that cheating can be enhanced in subjects who are given to read a written text passage stating that free will is an illusion [126]. This has been interpreted as an indication that the belief in free will decreases cheating, which is a meaningful statement only under the implicit assumption that our conscious beliefs can be causally effective in exerting an influence on the processes that occur in the physical world. Those who believe that free will is an illusion due to determinism have been fast to adopt an evolutionary explanation for the origin of the free will illusion, namely, we have evolved to believe in free will because this make us nicer people in a social context. The latter statement is demonstrably false, however, because conscious experiences are epiphenomenal in deterministic functional theories of the human mind $[1,37,38,127]$. The proof starts from the observation that conscious experiences do not enter directly into Hamilton's equations (9) of classical physics. Therefore, conscious experiences can be generated by the brain, but cannot change in any way the physical dynamics of the brain, which is already fully determined by the classical physical quantities that comprise the brain and enter directly into Hamilton's equations. In other words, to predict the future dynamics of a deterministic brain, we do not need to know whether it experiences something or not. Instead, we just compute numerically what the Hamilton's equations predict. Thus, the presence of conscious experiences in a deterministic physical world cannot have any physical manifestation, which means that consciousness can only be admitted as a causally ineffective epiphenomenon. Epiphenomenal beliefs cannot evolve through natural selection, which directly contradicts the claim that the belief in free 
will makes us nicer human beings in a social context. In essence, determinism forbids both free will and the evolutionary origin of illusionary belief in free will.

Free will cannot evolve in a deterministic world. Whether the physical world obeys deterministic or indeterministic physical laws is a universal statement. Physical laws do not evolve in time, which implies that free will is either allowed or forbidden at all times. Because natural selection operates only on physically possible variations, it follows that organisms cannot evolve free will in a deterministic physical world that forbids free will. Nevertheless, some 21st century philosophers have claimed that free will does evolve in a deterministic world. Daniel Dennett has proposed a re-interpretation of free will in terms of avoiding undesirable consequences [33]. For example, a conscious agent could contemplate that bumping into a stone laying on the road may break his leg and take avoiding action to circumvent the stone. At first glance, such a definition agrees with the meaning of free will, namely, avoiding an undesirable outcome that has non-zero probability is a beneficial thing to do and certainly we will exercise our free will in the act of choosing the beneficial outcome. In deterministic physical theories, however, the only allowable probabilities are zero or one. Therefore, given a classical model of the physical state of the world including ourselves, there are only two possible cases: If the undesirable outcome occurs with probability of one, then it is unavoidable and nothing that we can do will make any difference. Similarly, if the undesirable outcome occurs with zero probability, then it is impossible and again nothing that we can do will make any difference. For example, consider the ancient Greeks who prayed to Zeus, the god of the sky and thunder, not to strike them with a bolt of lightning. Because Zeus does not exist, there is a zero probability that Zeus strikes anybody with a lightning. Consequently, the prayers of ancient Greeks cannot be beneficial to avoid something that is impossible to occur anyway, and it would be meaningless to attribute free will to those who prayed based on the fact that they have avoided the impossible wrath of Zeus. Exactly the same will be the analysis of avoiding the stone laying on the road in classical physics. Superficially, the stone is a physical object and one could easily imagine how bumping into a stone could break one's leg. In deterministic classical physics, however, bumping into the stone given the exact physical state of the world is either a solution of Hamilton's equations (9), hence unavoidable, or it is not a solution, hence impossible. If we were to grant free will to physical processes that avoid the impossible, then by definition all physical processes would possess free will and the concept of free will would mean nothing. Noteworthy, our quantitative measure of free will (7) is mathematically impervious to superfluous addition of impossible outcomes due to the identity $0=0 \log _{2} 0$.

The randomness problem. A common but misleading argument against the relevance of quantum physics for the existence of free will is to claim that if our choices were performed by flipping a fair coin, then the resulting actions will be random manifestations of chance or luck, hence they will be incompatible with free will. The mistake in such argument is the conflation of external and internal causes into a single category, namely, free will is defined by an inherent propensity to perform choices, whereas the fair coin is an external agent. Indeed, if a person flips a coin and executes the outcome chosen by the coin, then the person manifests zero free will as the act of copying the result from the coin is perfectly deterministic. The same will be true if one films on a tape the behavior of a person endowed with free will and then asks another person to copy the recorded behavior. The second person manifests zero free will in the act of copying the previously recorded behavior. A possible attempt to fix the argument could be to insist that if the fair coin performs genuine unbiased choices, then we should attribute free will to the fair coin itself. But the answer is that this is exactly what we do, namely, if we attribute free will to the brain by virtue of the physical laws, then we also attribute free will to the constituent physical particles that build up the brain. The free will does not pop in and out of existence in violation of physical laws. In fact, in the evolutionary history of the animal nervous systems, the narrative is reversed, that is the brain possesses free will exactly because the physical components from which it is built possess free will [1]. The latter position has been previously advocated by Conway and Kochen who formalized their reasoning into a set of axioms from which they derived the so-called free will theorem [128, 129]. 


\section{References}

[1] D. D. Georgiev. Quantum Information and Consciousness: A Gentle Introduction. CRC Press, Boca Raton, 2017. doi:10.1201/9780203732519.

[2] R. Kane. The Significance of Free Will. Oxford University Press, Oxford, 1996.

[3] R. Kane. Responsibility, luck, and chance: reflections on free will and indeterminism. Journal of Philosophy 1999; 96(5):217-240. doi:10.2307/2564666.

[4] R. Kane. A Contemporary Introduction to Free Will. Fundamentals of Philosophy. Oxford University Press, Oxford, 2005.

[5] J.-P. Sartre. The Philosophy of Existentialism. Philosophical Library, New York, 1965.

[6] J.-P. Sartre. Existentialism is a Humanism. Yale University Press, New Haven, Connecticut, 2007.

[7] J. Rawls. Justice as fairness. The Philosophical Review 1958; 67(2):164-194. doi:10.2307/2182612.

[8] M. Lazerowitz, A. Ambrose. Free will. Crítica: Revista Hispanoamericana de Filosofía 1984; 16(48):3-17. doi:10.22201/ iifs.18704905e.1984.551.

[9] L. Susskind, G. Hrabovsky. The Theoretical Minimum: What You Need to Know to Start Doing Physics. Basic Books, New York, 2013.

[10] A. Schopenhauer. Prize essay on the freedom of the will. in: C. Janaway (Ed.), The Two Fundamental Problems of Ethics. The Cambridge Edition of the Works of Schopenhauer. Cambridge University Press, Cambridge, 1841. pp. 31112. doi:10.1017/cbo9780511581298.011.

[11] D. M. Wegner. The Illusion of Conscious Will. Bradford Books. MIT Press, Cambridge, Massachusetts, 2003.

[12] S. Nichols. Is free will an illusion?. Scientific American Mind 2011; 22(5):18-19.

[13] S. Harris. Free Will. Free Press, New York, 2012.

[14] R. Kane. The dual regress of free will and the role of alternative possibilities. Philosophical Perspectives 2000; 14:57-79. doi:10.1111/0029-4624.34.s14.4.

[15] R. Kane. Libertarianism. Philosophical Studies: An International Journal for Philosophy in the Analytic Tradition 2009; 144(1):35-44. doi:10.1007/s11098-009-9365-y.

[16] R. Kane. Acting 'of one's own free will': modern reflections on an ancient philosophical problem. Proceedings of the Aristotelian Society 2014; 114:35-55. doi:10.1111/j.1467-9264.2014.00363.x.

[17] C. E. Shannon. A mathematical theory of communication. The Bell System Technical Journal 1948; 27(3):379-423. doi:10.1002/j.1538-7305.1948.tb01338.x.

[18] C. E. Shannon. A mathematical theory of communication. The Bell System Technical Journal 1948; 27(4):623-656. doi: 10.1002/j.1538-7305.1948.tb00917.x.

[19] T. M. Cover, J. A. Thomas. Elements of Information Theory. 2nd Edition. Wiley Series in Telecommunications and Signal Processing. Wiley-Interscience, Hoboken, New Jersey, 2006.

[20] S. Kullback, R. A. Leibler. On information and sufficiency. Annals of Mathematical Statistics 1951; 22(1):79-86. doi: 10.1214/aoms/1177729694.

[21] S. Kullback. The Kullback-Leibler distance. The American Statistician 1987; 41(4):340-341. doi:10.1080/00031305. 1987.10475510.

[22] C. Adami. What is information?. Philosophical Transactions. Series A, Mathematical, Physical, and Engineering Sciences 2016; 374(2063):20150230. doi:10.1098/rsta.2015.0230.

[23] M. Gell-Mann, S. Lloyd. Information measures, effective complexity, and total information. Complexity 1996; 2(1):44-52. doi:10.1002/(SICI) 1099-0526(199609/10) $2: 1<44::$ AID-CPLX10>3.0.CO;2-X.

[24] D. Strauch. Classical Mechanics: An Introduction. Springer, Berlin, 2009. doi:10.1007/978-3-540-73616-5.

[25] P. Eastman. Introduction to Statistical Mechanics. Stanford University, Stanford, California, 2015. https://github. com/peastman/statmech.

[26] A. C. Scott. The Nonlinear Universe. Chaos, Emergence, Life. The Frontiers Collection. Springer, Berlin, 2007. doi: 10.1007/978-3-540-34153-6.

[27] R. Ramaswamy. Aspects of chaos in conservative dynamical systems. Current Science 1984; 53(12):619-626.

[28] E. N. Lorenz. Deterministic nonperiodic flow. Journal of the Atmospheric Sciences 1963; 20(2):130-141. doi:10.1175/ $1520-0469$ (1963) $020<0130: \mathrm{DNF}>2.0 . \mathrm{CO} ; 2$.

[29] E. N. Lorenz. The predictability of hydrodynamic flow. Transactions of the New York Academy of Sciences 1963; 25(4 Series II):409-432. doi:10.1111/j.2164-0947.1963.tb01464.x.

[30] S. W. Hawking, L. Mlodinow. The Grand Design: New Answers to the Ultimate Questions of Life. Bantam Press, New York, 2010.

[31] M. W. Hirsch, S. Smale, R. L. Devaney. Differential Equations, Dynamical Systems, and an Introduction to Chaos. 3rd Edition. Academic Press, Amsterdam, 2013. doi:10.1016/c2009-0-61160-0.

[32] B. Russell. Why I am not a Christian: and Other Essays on Religion and Related Subjects. Routledge, London, 2005. doi: $10.4324 / 9781315099552$.

[33] D. C. Dennett. Freedom Evolves. Penguin, New York, 2004.

[34] J. Locke. An Essay Concerning Human Understanding. J. F. Dove, London, 1828. https://archive.org/details/ anessayconcernio0unkngoog/.

[35] E. Schrödinger. Collected Papers on Wave Mechanics. Blackie \& Son, London, 1928.

[36] M. Hayashi, S. Ishizaka, A. Kawachi, G. Kimura, T. Ogawa. Introduction to Quantum Information Science. Graduate Texts in Physics. Springer, Berlin, 2015. doi:10.1007/978-3-662-43502-1. 
[37] D. D. Georgiev. Inner privacy of conscious experiences and quantum information. Biosystems 2020; 187:104051. doi: 10.1016/j.biosystems.2019.104051.

[38] D. D. Georgiev. Quantum information theoretic approach to the mind-brain problem. Progress in Biophysics and Molecular Biology 2020; 158:16-32. doi:10.1016/j.pbiomolbio.2020.08.002.

[39] D. D. Georgiev. Quantum information in neural systems. Symmetry 2021; 13(5):773. doi:10.3390/sym13050773.

[40] D. D. Georgiev, E. Cohen. Probing finite coarse-grained virtual Feynman histories with sequential weak values. Physical Review A 2018; 97(5):052102. doi:10.1103/PhysRevA.97.052102.

[41] D. Georgiev, L. Bello, A. Carmi, E. Cohen. One-particle and two-particle visibilities in bipartite entangled Gaussian states. Physical Review A 2021; 103(6):062211. doi:10.1103/PhysRevA.103.062211.

[42] P. A. M. Dirac. A new notation for quantum mechanics. Mathematical Proceedings of the Cambridge Philosophical Society 1939; 35(3):416-418. doi:10.1017/S0305004100021162.

[43] P. A. M. Dirac. The Principles of Quantum Mechanics. 4th Edition. Oxford University Press, Oxford, 1967.

[44] M. L. Bhaumik. How Dirac's seminal contributions pave the way for comprehending nature's deeper designs. Quanta $2019 ; 8(1): 88-100$. doi:10.12743/quanta.v8i1.96.

[45] Y. M. Berezansky, Z. G. Sheftel, G. F. Us. Functional Analysis. Vol. I. Vol. 85 of Operator Theory: Advances and Applications. Birkhäuser, Basel, 1996. doi:10.1007/978-3-0348-9185-1.

[46] M. Born. Statistical interpretation of quantum mechanics. Science 1955; 122(3172):675-679. doi:10.1126/science.122. 3172.675.

[47] P. Busch, P. Lahti, J.-P. Pellonpää, K. Ylinen. Quantum Measurement. Theoretical and Mathematical Physics. Springer, Cham, 2016. doi:10.1007/978-3-319-43389-9.

[48] M. Fayngold, V. Fayngold. Quantum Mechanics and Quantum Information. Wiley-VCH, Weinheim, Germany, 2013.

[49] L. Susskind, A. Friedman. Quantum Mechanics: The Theoretical Minimum. What You Need to Know to Start Doing Physics. Basic Books, New York, 2014.

[50] A. S. Holevo. Statistical Structure of Quantum Theory. Lecture Notes in Physics. Springer, 2001. doi:10.1007/3-54044998-1.

[51] W. G. Strang. Introduction to Linear Algebra. 5th Edition. Wellesley-Cambridge Press, Wellesley, Massachusetts, 2016.

[52] W. Gerlach, O. Stern. Der experimentelle Nachweis der Richtungsquantelung im Magnetfeld. Zeitschrift für Physik 1922; 9(1):349-352. doi:10.1007/bf01326983.

[53] S. Cruz-Barrios, J. Gómez-Camacho. Semiclassical description of Stern-Gerlach experiments. Physical Review A 2000; 63(1):012101. doi:10.1103/PhysRevA.63.012101.

[54] G. Potel, F. Barranco, S. Cruz-Barrios, J. Gómez-Camacho. Quantum mechanical description of Stern-Gerlach experiments. Physical Review A 2005; 71(5):052106. doi:10.1103/PhysRevA.71.052106.

[55] K. Matsuno, R. C. Paton. Is there a biology of quantum information?. Biosystems 2000; 55(1):39-46. doi:10.1016/S03032647 (99) $00081-7$

[56] K. Matsuno. Forming and maintaining a heat engine for quantum biology. Biosystems 2006; 85(1):23-29. doi:10.1016/ j.biosystems. 2006.02.002.

[57] A. V. Melkikh. Thinking as a quantum phenomenon. Biosystems 2019; 176:32-40. doi:10.1016/j.biosystems.2018. 12.007.

[58] C. C. Chancey, S. A. George, P. J. Marshall. Calculations of quantum tunnelling between closed and open states of sodium channels. Journal of Biological Physics 1992; 18(4):307-321. doi:10.1007/bf 00419427.

[59] A. Vaziri, M. B. Plenio. Quantum coherence in ion channels: resonances, transport and verification. New Journal of Physics 2010; 12(8):085001. doi:10.1088/1367-2630/12/8/085001.

[60] A. M. Kariev, M. E. Green. Quantum calculation of proton and other charge transfer steps in voltage sensing in the Kv1.2 channel. Journal of Physical Chemistry B 2019; 123(38):7984-7998. doi:10.1021/acs.jpcb.9b05448.

[61] A. M. Kariev, M. E. Green. Quantum calculations on ion channels: why are they more useful than classical calculations, and for which processes are they essential?. Symmetry 2021; 13(4):655. doi:10.3390/sym13040655.

[62] D. D. Georgiev, J. F. Glazebrook. The quantum physics of synaptic communication via the SNARE protein complex. Progress in Biophysics and Molecular Biology 2018; 135:16-29. doi:10.1016/j.pbiomolbio.2018.01.006.

[63] D. D. Georgiev, S. K. Kolev, E. Cohen, J. F. Glazebrook. Computational capacity of pyramidal neurons in the cerebral cortex. Brain Research 2020; 1748:147069. doi:10.1016/j.brainres.2020.147069.

[64] M.-H. Kim, G.-L. Li, H. von Gersdorff. Single $\mathrm{Ca}^{2+}$ channels and exocytosis at sensory synapses. Journal of Physiology 2013; 591(13):3167-3178. doi:10.1113/jphysiol.2012.249482.

[65] J. H. Singer. Multivesicular release and saturation of glutamatergic signalling at retinal ribbon synapses. Journal of Physiology 2007; 580(1):23-29. doi:10.1113/jphysiol.2006.125302.

[66] E. Glowatzki, P. A. Fuchs. Transmitter release at the hair cell ribbon synapse. Nature Neuroscience 2002; 5:147. doi: 10.1038/nn796.

[67] J. Magistretti, P. Spaiardi, S. L. Johnson, S. Masetto. Elementary properties of Ca ${ }^{2+}$ channels and their influence on multivesicular release and phase-locking at auditory hair cell ribbon synapses. Frontiers in Cellular Neuroscience 2015; 9:123. doi:10.3389/fncel.2015.00123.

[68] M. Kuno, S. A. Turkanis, J. N. Weakly. Correlation between nerve terminal size and transmitter release at the neuromuscular junction of the frog. Journal of Physiology 1971; 213(3):545-556. doi:10.1113/jphysiol.1971.sp009399.

[69] S. Rudolph, M.-C. Tsai, H. von Gersdorff, J. I. Wadiche. The ubiquitous nature of multivesicular release. Trends in Neurosciences 2015; 38(7):428-438. doi:10.1016/j.tins.2015.05.008.

[70] C. Pulido, A. Marty. Quantal fluctuations in central mammalian synapses: functional role of vesicular docking sites. Physiological Reviews 2017; 97(4):1403-1430. doi:10.1152/physrev.00032.2016. 
[71] F. Beck, J. C. Eccles. Quantum aspects of brain activity and the role of consciousness. Proceedings of the National Academy of Sciences 1992; 89(23):11357-11361. doi:10.1073/pnas.89.23.11357.

[72] O. Arancio, H. Korn, A. Gulyas, T. Freund, R. Miles. Excitatory synaptic connections onto rat hippocampal inhibitory cells may involve a single transmitter release site. Journal of Physiology 1994; 481(2):395-405. doi:10.1113/jphysiol. 1994 .sp020448.

[73] V. Matveev, X. J. Wang. Implications of all-or-none synaptic transmission and short-term depression beyond vesicle depletion: a computational study. Journal of Neuroscience 2000; 20(4):1575-1588. doi:10.1523/jneurosci.20-0401575.2000 .

[74] E. Hanse, B. Gustafsson. Vesicle release probability and pre-primed pool at glutamatergic synapses in area CA1 of the rat neonatal hippocampus. Journal of Physiology 2001; 531(2):481-493. doi:10.1111/j.1469-7793.2001.0481i.x.

[75] C. F. Stevens, Y. Wang. Facilitation and depression at single central synapses. Neuron 1995; 14(4):795-802. doi: 10.1016/0896-6273(95) 90223-6.

[76] L. E. Dobrunz, C. F. Stevens. Heterogeneity of release probability, facilitation, and depletion at central synapses. Neuron 1997; 18(6):995-1008. doi:10.1016/S0896-6273(00)80338-4.

[77] T. Weber, B. V. Zemelman, J. A. McNew, B. Westermann, M. Gmachl, F. Parlati, T. H. Söllner, J. E. Rothman. SNAREpins: minimal machinery for membrane fusion. Cell 1998; 92(6):759-772. doi:10.1016/S0092-8674(00)81404-X.

[78] J. Han, K. Pluhackova, R. A. Böckmann. The multifaceted role of SNARE proteins in membrane fusion. Frontiers in Physiology 2017; 8:5. doi:10.3389/fphys.2017.00005.

[79] D. D. Georgiev, J. F. Glazebrook. On the quantum dynamics of Davydov solitons in protein $\alpha$-helices. Physica A: Statistical Mechanics and its Applications 2019; 517:257-269. doi:10.1016/j.physa.2018.11.026.

[80] D. D. Georgiev, J. F. Glazebrook. Quantum tunneling of Davydov solitons through massive barriers. Chaos, Solitons and Fractals 2019; 123:275-293. doi:10.1016/j.chaos.2019.04.013.

[81] D. D. Georgiev, J. F. Glazebrook. Quantum transport and utilization of free energy in protein $\alpha$-helices. Advances in Quantum Chemistry 2020; 82:253-300. doi:10.1016/bs.aiq.2020.02.001.

[82] D. D. Georgiev, J. F. Glazebrook. Launching of Davydov solitons in protein $\alpha$-helix spines. Physica E: Low-dimensional Systems and Nanostructures 2020; 124:114332. doi:10.1016/j.physe.2020.114332.

[83] E. R. Chapman. Synaptotagmin: a $\mathrm{Ca}^{2+}$ sensor that triggers exocytosis?. Nature Reviews Molecular Cell Biology 2002; 3(7):498-508. doi:10.1038/nrm855.

[84] Q. Zhou, Y. Lai, T. Bacaj, M. Zhao, A. Y. Lyubimov, M. Uervirojnangkoorn, O. B. Zeldin, A. S. Brewster, N. K. Sauter, A. E. Cohen, S. M. Soltis, R. Alonso-Mori, M. Chollet, H. T. Lemke, R. A. Pfuetzner, U. B. Choi, W. I. Weis, J. Diao, T. C. Südhof, A. T. Brunger. Architecture of the synaptotagmin-SNARE machinery for neuronal exocytosis. Nature $2015 ; \mathbf{5 2 5}(7567): 62-67$. doi:10.1038/nature14975.

[85] Q. Zhou, P. Zhou, A. L. Wang, D. Wu, M. Zhao, T. C. Südhof, A. T. Brunger. The primed SNARE-complexinsynaptotagmin complex for neuronal exocytosis. Nature 2017; 548(7668):420-425. doi:10.1038/nature23484.

[86] V. Braitenberg, A. Schüz. Cortex: Statistics and Geometry of Neuronal Connectivity. 2nd Edition. Springer, Berlin, 1998. doi:10.1007/978-3-662-03733-1.

[87] F. A. C. Azevedo, L. R. B. Carvalho, L. T. Grinberg, J. M. Farfel, R. E. L. Ferretti, R. E. P. Leite, W. J. Filho, R. Lent, S. Herculano-Houzel. Equal numbers of neuronal and nonneuronal cells make the human brain an isometrically scaled-up primate brain. Journal of Comparative Neurology 2009; 513(5):532-541. doi:10.1002/cne.21974.

[88] R. Nieuwenhuys. The neocortex. Anatomy and Embryology 1994; 190(4):307-337. doi:10.1007/bf00187291.

[89] F. R. Walther. Flight behaviour and avoidance of predators in Thomson's gazelle (Gazella Thomsoni Guenther 1884). Behaviour 1969; 34(3):184-220. doi:10.1163/156853969x00053.

[90] L. Hudson. Contrary Imaginations: A Psychological Study of the English Schoolboy. Pelican Books. Penguin Books, Harmondsworth, 1974.

[91] A. Cardoso, T. Veale, G. A. Wiggins. Converging on the divergent: The history (and future) of the international joint workshops in computational creativity. AI Magazine 2009; 30(3):15-22.

[92] M. A. Runco, S. R. Pritzker. Encyclopedia of Creativity, Two-Volume Set. 3rd Edition. Academic Press, Oxford, 2020.

[93] G. V. Georgiev, D. D. Georgiev. Enhancing user creativity: semantic measures for idea generation. Knowledge-Based Systems 2018; 151:1-15. doi:10.1016/j.knosys.2018.03.016.

[94] G. M. Morriss-Kay. The evolution of human artistic creativity. Journal of Anatomy 2010; 216(2):158-176. doi:10.1111/ j.1469-7580.2009.01160.x.

[95] D. Hodgson, J. Verpooten. The evolutionary significance of the arts: exploring the by-product hypothesis in the context of ritual, precursors, and cultural evolution. Biological Theory 2015; 10(1):73-85. doi:10.1007/s13752-014-0182-y.

[96] R. Kane. Free will: the elusive ideal. Philosophical Studies: An International Journal for Philosophy in the Analytic Tradition 1994; 75(1-2):25-60. doi:10.1007/BF00989880.

[97] R. Kane. Freedom, responsibility, and will-setting. Philosophical Topics 1996; 24(2):67-90. doi:10.5840/ philtopics199624210.

[98] R. Kane. Free will and responsibility: ancient dispute, new themes. The Journal of Ethics 2000; 4(4):315-322. doi: 10.1023/A: 1026729231975

[99] R. Kane. Moral responsibility, reactive attitudes and freedom of will. The Journal of Ethics 2016; 20(1-3):229-246. doi:10.1007/s10892-016-9234-9.

[100] C. Darrow, W. Durant. Debate: Is Man a Machine? Clarence Darrow, Affirmative; Dr. Will Durant, Negative. The League for Public Discussion, New York, 1927.

[101] C. Darrow. The Story of My Life. Charles Scribner's Sons, New York, 1932.

[102] J. Trommershäuser, R. Schneggenburger, A. Zippelius, E. Neher. Heterogeneous presynaptic release probabilities: func- 
tional relevance for short-term plasticity. Biophysical journal 2003; 84(3):1563-1579. doi:10.1016/s0006-3495(03) 74967-4.

[103] T. Branco, K. Staras. The probability of neurotransmitter release: variability and feedback control at single synapses. Nature Reviews Neuroscience 2009; 10(5):373-383. doi:10.1038/nrn2634.

[104] C. Körber, T. Kuner. Molecular machines regulating the release probability of synaptic vesicles at the active zone. Frontiers in Synaptic Neuroscience 2016; 8:5-5. doi:10.3389/fnsyn.2016.00005.

[105] H. Taschenberger, A. Woehler, E. Neher. Superpriming of synaptic vesicles as a common basis for intersynapse variability and modulation of synaptic strength. Proceedings of the National Academy of Sciences 2016; 113(31):E4548-E4557. doi:10.1073/pnas.1606383113.

[106] M. Volgushev, I. Kudryashov, M. Chistiakova, M. Mukovski, J. Niesmann, U. T. Eysel. Probability of transmitter release at neocortical synapses at different temperatures. Journal of Neurophysiology 2004; 92(1):212-220. doi:10.1152/jn. 01166.2003

[107] N. D. Volkow, T.-K. Li. Drug addiction: the neurobiology of behaviour gone awry. Nature Reviews Neuroscience 2004; 5(12):963-970. doi:10.1038/nrn1539.

[108] C. Lüscher, T. W. Robbins, B. J. Everitt. The transition to compulsion in addiction. Nature Reviews Neuroscience 2020; 21(5):247-263. doi:10.1038/s41583-020-0289-z.

[109] G. F. Koob. Neurobiology of opioid addiction: opponent process, hyperkatifeia, and negative reinforcement. Biological Psychiatry 2020; 87(1):44-53. doi:10.1016/j.biopsych.2019.05.023.

[110] B. J. Venton, A. T. Seipel, P. E. M. Phillips, W. C. Wetsel, D. Gitler, P. Greengard, G. J. Augustine, R. M. Wightman. Cocaine increases dopamine release by mobilization of a synapsin-dependent reserve pool. Journal of Neuroscience 2006; 26(12):3206-3209. doi:10.1523/jneurosci.4901-04.2006.

[111] S. A. Buck, M. M. Torregrossa, R. W. Logan, Z. Freyberg. Roles of dopamine and glutamate co-release in the nucleus accumbens in mediating the actions of drugs of abuse. FEBS Journal 2020; doi:10.1111/febs.15496.

[112] T. Branco, K. Staras, K. J. Darcy, Y. Goda. Local dendritic activity sets release probability at hippocampal synapses. Neuron 2008; 59(3):475-485. doi:10.1016/j.neuron.2008.07.006.

[113] V. N. Murthy, T. Schikorski, C. F. Stevens, Y. Zhu. Inactivity produces increases in neurotransmitter release and synapse size. Neuron 2001; 32(4):673-682. doi:10.1016/S0896-6273(01)00500-1.

[114] R. D. Lee. Rethinking the evolutionary theory of aging: transfers, not births, shape senescence in social species. Proceedings of the National Academy of Sciences 2003; 100(16):9637-9642. doi:10.1073/pnas.1530303100.

[115] P. C. Lee, V. Fishlock, C. E. Webber, C. J. Moss. The reproductive advantages of a long life: longevity and senescence in wild female African elephants. Behavioral Ecology and Sociobiology 2016; 70(3):337-345. doi:10.1007/s00265-0152051-5.

[116] L. A. Fairbanks. Vervet monkey grandmothers: interactions with infant grandoffspring. International Journal of Primatology 1988; 9(5):425-441. doi:10.1007/bf02736218.

[117] L. A. Fairbanks. Vervet monkey grandmothers: effects on mother-infant relationships. Behaviour 1988; 104(1-2):176-188. doi:10.1163/156853988x00665.

[118] M. Nakamichi, K. Onishi, K. Yamada. Old grandmothers provide essential care to their young granddaughters in a freeranging group of Japanese monkeys (Macaca fuscata). Primates 2010; 51(2):171-174. doi:10.1007/s10329-009-0177-7.

[119] M. Lahdenperä, K. U. Mar, V. Lummaa. Nearby grandmother enhances calf survival and reproduction in Asian elephants. Scientific Reports 2016; 6(1):27213. doi:10.1038/srep27213.

[120] J. E. Coxworth, P. S. Kim, J. S. McQueen, K. Hawkes. Grandmothering life histories and human pair bonding. Proceedings of the National Academy of Sciences 2015; 112(38):11806-11811. doi:10.1073/pnas.1599993112.

[121] S. Nattrass, D. P. Croft, S. Ellis, M. A. Cant, M. N. Weiss, B. M. Wright, E. Stredulinsky, T. Doniol-Valcroze, J. K. B. Ford, K. C. Balcomb, D. W. Franks. Postreproductive killer whale grandmothers improve the survival of their grandoffspring. Proceedings of the National Academy of Sciences 2019; 116(52):26669-26673. doi:10.1073/pnas.1903844116.

[122] K. Hawkes, J. F. O'Connell, N. G. Blurton Jones. Hadza women's time allocation, offspring provisioning, and the evolution of long postmenopausal life spans. Current Anthropology 1997; 38(4):551-577. doi:10.1086/204646.

[123] P. S. Kim, J. E. Coxworth, K. Hawkes. Increased longevity evolves from grandmothering. Proceedings of the Royal Society B: Biological Sciences 2012; 279(1749):4880-4884. doi:10.1098/rspb.2012.1751.

[124] D. A. Coall, R. Hertwig. Grandparental investment: past, present, and future. Behavioral and Brain Sciences 2010; 33(1):1-19. doi:10.1017/s0140525x09991105.

[125] H. G. Frankfurt. Alternate possibilities and moral responsibility. Journal of Philosophy 1969; 66(23):829-839. doi: 10.2307/2023833.

[126] K. D. Vohs, J. W. Schooler. The value of believing in free will: encouraging a belief in determinism increases cheating. Psychological Science 2008; 19(1):49-54. doi:10.1111/j.1467-9280.2008.02045.x.

[127] D. D. Georgiev. Quantum no-go theorems and consciousness. Axiomathes 2013; 23(4):683-695. doi:10.1007/s10516012-9204-1.

[128] J. H. Conway, S. B. Kochen. The free will theorem. Foundations of Physics 2006; 36(10):1441-1473. doi:10.1007/ s10701-006-9068-6.

[129] J. H. Conway, S. B. Kochen. The strong free will theorem. Notices of the AMS 2009; 56(2):226-232. 